Linköping Studies in Science and Technology

Dissertation, No. 2142

\title{
Multi-Cell Massive MIMO: \\ Power Control and Channel Estimation
}

Amin Ghazanfari
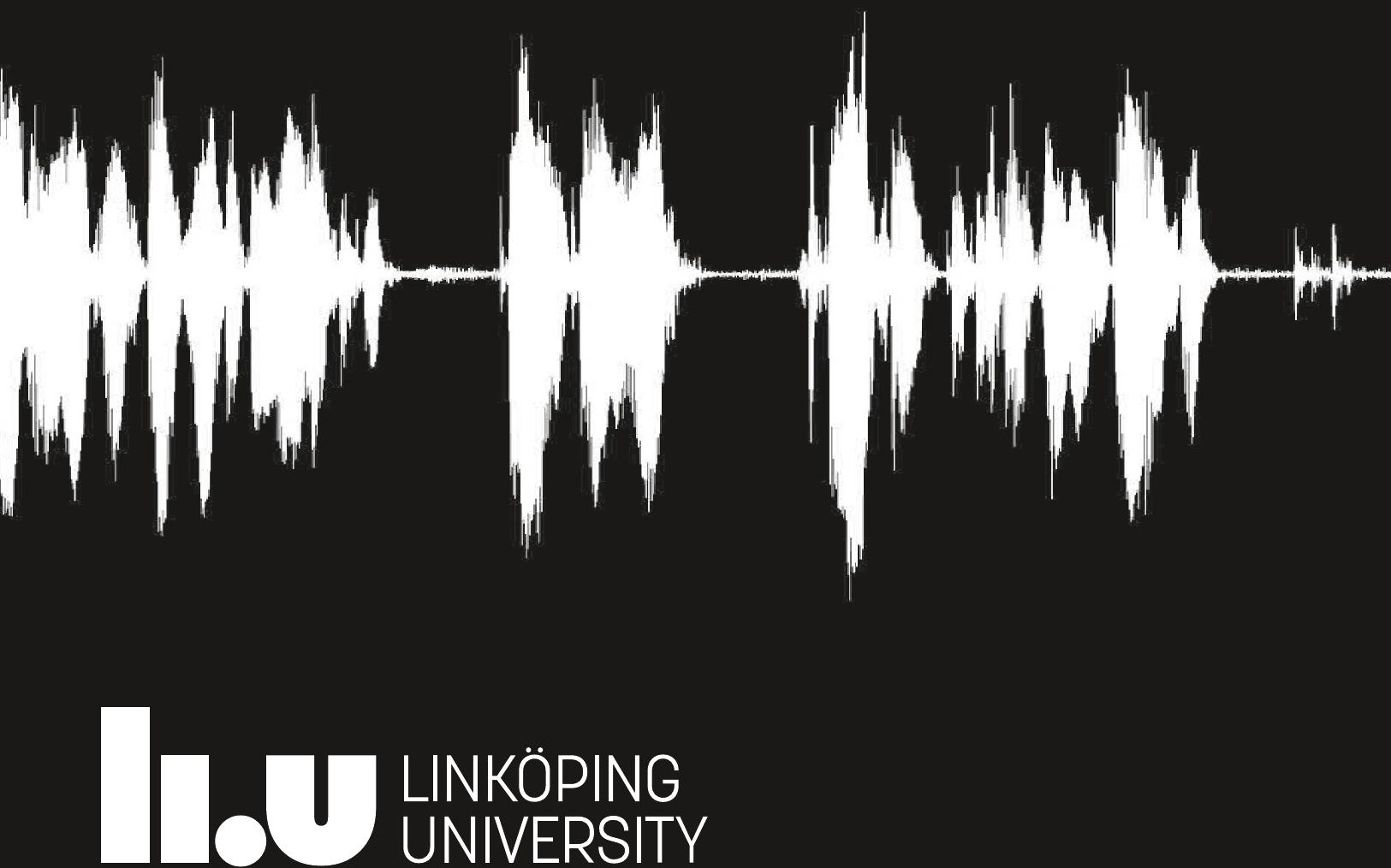

LINKÖPING
UNIVERSITY 



\section{Multi-Cell Massive MIMO: Power Control and Channel Estimation}

Amin Ghazanfari

\section{EOMMUNICATION SŶstems}

Division of Communication Systems

Department of Electrical Engineering (ISY)

Linköping University, 58183 Linköping, Sweden

www.commsys.isy.liu.se

Linköping 2021 
This is a Swedish Doctor of Philosophy thesis.

The Doctor of Philosophy degree comprises 240 ECTS credits of postgraduate studies.

(c) EY-No This work is licensed under a Creative Commons AttributionNonCommercial 4.0 International License.

https://creativecommons.org/licenses/by-nc/4.0/

Multi-Cell Massive MIMO: Power Control and Channel Estimation

(c) 2021 Amin Ghazanfari, unless otherwise stated.

ISBN 978-91-7929-651-3

ISSN 0345-7524

Printed in Sweden by LiU-Tryck, Linköping 2021 


To my love, Nastaran 



\section{Abstract}

Cellular network operators have witnessed significant growth in data traffic in the past few decades. This growth occurs due to the increase in the number of connected mobile devices, and further, the emerging mobile applications developed for rendering video-based on-demand services. As the available frequency bandwidth for cellular communication is limited, significant efforts are dedicated to improving the utilization of available spectrum and increasing the system performance with the aid of new technologies. Third-generation (3G) and fourth-generation (4G) mobile communication networks were designed to facilitate high data traffic in cellular networks in past decades. Nevertheless, there is still a requirement for new cellular network technologies to accommodate the ever-growing data traffic demand. The fifth-generation $(5 \mathrm{G})$ is the latest generation of mobile communication systems deployed and implemented around the world. Its objective is to meet the tremendous ongoing increase in the data traffic requirements in cellular networks.

Massive MIMO (multiple-input-multi-output) is one of the backbone technologies in 5G networks. Massive MIMO originated from the concept of multi-user MIMO. It consists of base stations (BSs) implemented with a large number of antennas to increase the signal strengths via adaptive beamforming and concurrently serving many users on the same time-frequency blocks. With Massive MIMO technology, there is a notable enhancement of both sum spectral efficiency (SE) and energy efficiency (EE) in comparison with conventional MIMO-based cellular networks. Resource allocation is an imperative factor to exploit the specified gains of Massive MIMO. It corresponds to efficiently allocating resources in the time, frequency, space, and power domains for cellular communication. Power control is one of the resource allocation methods of Massive MIMO networks to deliver high spectral and energy efficiency. Power control refers to a scheme that allocates transmit powers to the data transmitters such that the system maximizes some desirable performance metric.

The first part of this thesis investigates reusing a Massive MIMO network's resources for direct communication of some specific user pairs known as deviceto-device (D2D) underlay communication. D2D underlay can conceivably increase 
the SE of traditional Massive MIMO networks by enabling more simultaneous transmissions on the same frequencies. Nevertheless, it adds additional mutual interference to the network. Consequently, power control is even more essential in this scenario than the conventional Massive MIMO networks to limit the interference caused by the cellular network and the D2D communication to enable their coexistence. We propose a novel pilot transmission scheme for D2D users to limit the interference on the channel estimation phase of cellular users compared with sharing pilot sequences for cellular and D2D users. We also introduce a novel pilot and data power control scheme for D2D underlaid Massive MIMO networks. This method aims to assure that the D2D communication enhances the SE of the network compared to conventional Massive MIMO networks.

In the second part of this thesis, we propose a novel power control approach for multi-cell Massive MIMO networks. The proposed power control approach solves the scalability issue of two well-known power control schemes frequently used in the Massive MIMO literature, based on the network-wide max-min and proportional fairness performance metrics. We first identify the scalability issue of these existing approaches. Additionally, we provide mathematical proof for the scalability of our proposed method. Our scheme aims at maximizing the geometric mean of the per-cell max-min SE. To solve the optimization problem, we prove that it can be rewritten in a convex form and is solved using standard optimization solvers.

The final part of this thesis focuses on downlink channel estimation in a Massive MIMO network. In Massive MIMO networks, to fully benefit from large antennas at the BSs and perform resource allocation, the BS must have access to high-quality channel estimates that can be acquired via the uplink pilot transmission phase. Time-division duplex (TDD) based Massive MIMO relies on channel reciprocity for the downlink transmission. Thanks to the channel hardening in the Massive MIMO networks with ideal propagation conditions, users rely on the statistical knowledge of channels for decoding the data in the downlink. However, when the channel hardening level is low, using only the channel statistics causes fluctuations in the performance. We investigate how to improve the performance by empowering the user to estimate the downlink channel from downlink data transmissions utilizing a model-based and a data-driven approach instead of relying only on channel statistics. Furthermore, the performance of the proposed method is compared with solely relying on statistical knowledge. 


\section{Populärvetenskaplig Sammanfattning}

Mobiloperatörerna har upplevt en snabb tillväxt i datatrafik under de senaste decennierna. Denna tillväxt har skapats genom en ökning av antalet uppkopplade enheter samt nya mobilapplikationer såsom videobaserade on-demand-tjänster. Eftersom den tillgängliga frekvensbandbredden för mobilkommunikation är begränsad så är ökningar i prestanda nära kopplade till utvecklandet av ny teknik som förbättrar utnyttjandet av det tillgängliga spektrumet. Tredje generationens (3G) och fjärde generationens $(4 \mathrm{G})$ mobilteknik utformades för att möjliggöra de senaste decennierna ökning i datatrafik, men den ständigt växande efterfrågan på dataöverföring kräver utveckling av nya mobilnätstekniker. Den femte generationen $(5 \mathrm{G})$ av mobilnät håller på att byggas i många länder världen över och det finns många 5G-mobiler tillgängliga på marknaden. 5G är tänkt att tillgodose det växande behovet av datatrafik under det kommande decenniet.

En ny teknik som kallas Massiv MIMO (multiple-input-multiple-output) är en grundbult i 5G. Massiv MIMO-system består av basstationer med ett stort antal antenner som kan öka signalstyrkan hos mottagaren genom adaptiv lobformning och samtidigt betjäna många användare i varje tidsfrekvensblock. Denna teknik förväntas leverera både högre dataöverföringshastigheter och öka energieffektivitet jämfört med konventionella mobilnät. Resursallokering är en avgörande faktor för att uppnå dessa prestandavinster. Detta avser tilldelningen av radioresurser inom tids-, frekvens-, rums- och effektdomänerna till användarna i mobilnätet. Till exempel avser effektreglering design av algoritmer för att tilldela signaleffekter till datasändarna så att systemet maximerar något gemensamt önskvärt prestandamått, såsom hög genomsnittlig datahastighet eller energieffektivitet.

Den första delen av denna avhandling undersöker hur radioresurserna i ett mobilnät som är baserat på Massiv MIMO kan samtidigt användas för direktkommunikation mellan närliggande enheter, så kallad device-to-device (D2D)kommunikation. Genom att tillåta D2D-kommunikation så kan den totala dataöver- 
föringshastigheten potentiellt öka, eftersom det är fler samtidiga överföringar på samma tidsfrekvensblock. Nackdelen är att det finns ytterligare störningar mellan de samtidiga sändningarna i systemet. Följaktligen blir effektreglering ännu viktigare i detta scenario än i vanliga Massiv MIMO-system, för att begränsa störningarna och därigenom möjliggöra samexistens mellan mobilnätet och D2Dkommunikationen. I den här delen av avhandlingen föreslår vi nya effektregleringsalgoritmer för att säkerställa att den totala dataöverföringshastigheten ökar när D2D-kommunikationen tillåts.

I den andra delen av denna avhandling föreslår vi nya effektregleringsalgoritmer för stora mobilnät med många basstationer som använder Massiv MIMO-teknik. Målet är att adressera skalbarhetsbrister som finns i existerande algoritmer, som fungerar sämre ju större näten är. Vi studerar flera olika prestandamått som kan optimeras via effektreglering. Det första är att maximera den lägsta dataöverföringshastigheten bland användarna i hela nätet. Den andra metoden kallas proportionell rättvisa och maximerar dataöverföringshastigheterna bland användarna proportionellt mot deras respektive kanalkvalitet. Vi identifierar skalbarhetsbristerna hos dessa algoritmer och förslår nya varianter som bevisligen är skalbara. Vårt föreslagna mått är att maximera den levererade datahastigheten i varje cell och sedan balansera dessa värden mellan olika celler i mobilnätet.

Den sista delen av avhandlingen fokuserar på nedlänken i ett mobilnät med Massiv-MIMO teknik, där basstationerna sänder data till mobilerna. För att dra nytta av det stora antalet antenner måste basstationerna skatta de trådlösa kanalerna så att lobformningen kan adapteras efter dessa. Information om kanalerna kan förvärvas på basstationerna genom att låta mobilerna skicka kända upplänkssignaler. Basstationerna använder kanalinformationen för att lobforma. Även mobilerna behöver viss kanalkännedom för att kunna avkoda den data som mottas i nedlänken. Den vanligaste lösningen i Massiv MIMO är att låta mobilerna förlita sig på statistisk kunskap om de lobformade radiokanalernas beteende. Detta antagande är inte att föredra under praktiska förhållanden där det är stora fluktuationer i kanalerna, vilket leder till alltför låga dataöverföringshastigheter. I den här delen av avhandlingen undersöker vi hur man kan förbättra prestandan genom att extrahera den kanalinformationen som finns i de mottagna datasignalerna. Vi föreslår en modellbaserad lösning och en datadriven metod, samt demonstrerar deras respektive fördelar gentemot tidigare metoder som förlitar sig på statistisk kanalkunskap. 


\section{Contents}

$\begin{array}{ll}\text { Acknowledgements } & \text { ix }\end{array}$

List of Abbreviations $\quad$ xi

1 Introduction 1

1.1 Motivation ................... 1

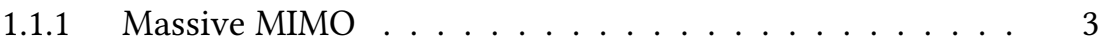

1.1.2 D2D Communication . . . . . . . . . . . . . 4

1.1.3 Topic and Motivation of the Thesis . . . . . . . . . . 4

1.2 Contributions of the Thesis . . . . . . . . . . . . . . 5

1.3 Papers Included in the Thesis . . . . . . . . . . . . . . . . 6

1.4 Papers Not Included in the Thesis . . . . . . . . . . . . . . 8

2 Massive MIMO $\quad 11$

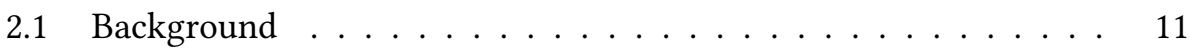

2.2 Key Properties of Massive MIMO . . . . . . . . . . . . . . . . . . 12

2.2.1 Favorable Propagation . . . . . . . . . . . . . 13

2.2.2 Channel Hardening . . . . . . . . . . . . . . . . . 14

2.2 .3 Duplexing Protocol . . . . . . . . . . . . . . . . 14

2.3 System Model . . . . . . . . . . . . . . . . . . . . . . . . 16

2.4 Channel Estimation . . . . . . . . . . . . . . . . 17

2.5 Data Transmission . . . . . . . . . . . . . . . . . . 19

3 Optimization Approaches 23

3.1 Convex Optimization ................. . . 24

3.2 Linear Programming . . . . . . . . . . . . . . 25

3.3 Epigraph Form . . . . . . . . . . . . . . . 25

3.4 Geometric Programming . . . . . . . . . . . . . 26

3.5 Signomial Programming ................ 27 
4 Power Control Schemes For Massive MIMO 29

4.1 Research Problems on Power Control . . . . . . . . . . . . . . 31

4.2 Max-min Fairness . . . . . . . . . . . . . . . . . . . 33

4.3 Proportional Fairness . . . . . . . . . . . . . . . . . 34

4.4 Use Cases . . . . . . . . . . . . . . . . . . . . . . . . 36

4.4.1 D2D Underlay Communications . . . . . . . . . . . 36

4.4.2 Scalability Issue for Power Control in Multi-cell Massive MIMO . . . . . . . . . . . . . . . . . . . . . 38

5 Neural Networks 39

5.1 Basics of Deep Learning . . . . . . . . . . . . . . . . . . 41

5.2 DNN for Channel Estimation . . . . . . . . . . . . . . . 44

$\begin{array}{ll}\text { Bibliography } & 46\end{array}$

Included Papers $\quad 56$

A Optimized Power Control for Massive MIMO with Underlaid D2D $\begin{array}{ll}\text { Communications } & 57\end{array}$

1 Introduction . . . . . . . . . . . . . . . . . . 59

$1.1 \quad$ Contributions of the Paper . . . . . . . . . . . 61

2 System Model . . . . . . . . . . . . . . . . . . 62

$2.1 \quad$ Uplink Data Transmission . . . . . . . . . . . . . 63

3 Analysis of Spectral Efficiency . . . . . . . . . . . . . . . 64

3.1 Pilot Transmission and Channel Estimation . . . . . . . . 64

3.2 Spectral Efficiency With MR Processing . . . . . . . . . 67

3.3 Spectral Efficiency With Zero-Forcing Processing . . . . . 68

3.4 Spectral Efficiency of D2D Communication . . . . . . 71

4 Optimization of Power Allocation . . . . . . . . . . . . 73

$4.1 \quad$ Data Power Control _. . . . . . . . . . . . . . . 74

4.2 Joint Pilot and Data Power Control for MR Processing . . 77

4.3 Joint Pilot and Data Power Control for ZF Processing . . . 78

5 Numerical Analysis . . . . . . . . . . . . . . . . . . 84

$5.1 \quad$ Optimize Data Power Control . . . . . . . . . . . . 85

$5.2 \quad$ Optimized Joint Pilot and Data Power Control . . . . . . . 87

6 Conclusion ...................... 91

$7 \quad$ Appendix ............................ 91

7.1 Tightness of the Approximate SE for D2D Communication 91

7.2 Basics of Geometric Programming . . . . . . . . 92 
7.3 Effect of D2D Distance on SE . . . . . . . . . . . 93

7.4 Effect of ZF Processing on Data Power Coefficients . . . 93

B Enhanced Fairness and Scalability of Power Control Schemes in Multi-cell Massive MIMO $\quad 99$

1 Introduction . . . . . . . . . . . . . . . . . 101

1.1 Related Works and Contributions . . . . . . . . . . . . 102

2 System Model . . . . . . . . . . . . . . . . . . . . . . . 105

3 Problem Formulation . . . . . . . . . . . . . . . . . . . . . 109

3.1 Proposed: Geometric-Mean Per-Cell Max-Min Fairness . . 109

3.2 Network-Wide Max-Min Fairness . . . . . . . . . . . . 111

3.3 Network-Wide Proportional Fairness . . . . . . . . . . . 113

4 Solutions to the Proposed Problems . . . . . . . . . . . . . . . 114

4.1 Per-Cell MMF Approximate Solution . . . . . . . . . . 116

4.2 Solution Approach for NW-MMF and NW-PF . . . . . . 117

5 Other Channel Models . . . . . . . . . . . . . . . . . . . . . . 118

6 Numerical Analysis . . . . . . . . . . . . . . . . . . . . . 120

7 Conclusion . . . . . . . . . . . . . . . . . . . . 127

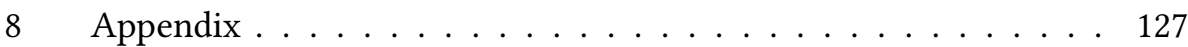

$8.1 \quad$ Proof of Lemma $3 \ldots \ldots$. . . . . . . . . . . . . 127

$8.2 \quad$ Power Budget Effects . . . . . . . . . . . . . . . . . . . 129

C Model-based and Data-driven Approaches for Downlink Massive MIMO Channel Estimation $\quad 135$

1 Introduction . . . . . . . . . . . . . . . . . . . . 137

2 System Model . . . . . . . . . . . . . . . . . . . . . . . . 140

$2.1 \quad$ Uplink Pilot Training . . . . . . . . . . . . . . . . . . . 141

2.2 Downlink Data Transmission . . . . . . . . . . . . . 143

3 Model-based Estimation of the Effective Downlink Channel Gain 145

3.1 Proposed Blind Channel Estimation with an Arbitrary Precoding Technique . . . . . . . . . . . . . . . 145

3.2 Downlink Channel Estimation With Linear Precoding Techniques . . . . . . . . . . . . . . . . . . . 148

4 Ergodic SE \& Asymptotic Analysis . . . . . . . . . . . . . . . . . . 149

$4.1 \quad$ Ergodic SE . . . . . . . . . . . . . . . . . 150

4.2 Asymptotic Analysis . . . . . . . . . . . . . . . . 152

5 Data-driven Approach for Downlink Massive MIMO Channel Estimation . . . . . . . . . . . . . . . . . . . . 154

6 Numerical Results . . . . . . . . . . . . . . . . . . . . . . 157

7 Conclusion . . . . . . . . . . . . . . . . . . . . . . 164 


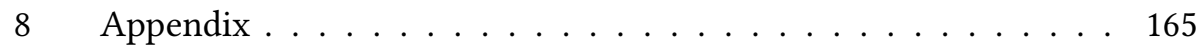

$8.1 \quad$ Proof of Lemma $2 \ldots \ldots \ldots \ldots$

$8.2 \quad$ Proof of Lemma $3 \ldots \ldots \ldots$ 


\section{Acknowledgements}

Destiny brought the boy who started school in an inferior condition in the Airforce military base in Chabahar, IRAN, to become a Ph.D. candidate in Sweden. It has been a long journey that allowed me to learn from all my teachers and mentors. I am incredibly thankful to any single one of them. They have helped, encouraged, supported, and guided me in so many ways.

First and foremost, I would like to express my deepest gratitude to my principal supervisor Professor Emil Björnson, for his patient guidance, enthusiastic encouragement, and beneficial critiques of my research. He always helped me with his advice, insightful comments, helpful feedback, and suggestions on my research problems. I would also like to express my sincere thanks to my co-supervisor, Professor Erik G. Larsson, for offering me the opportunity to join the Division of Communication Systems and for his constructive comments and discussions on my research problems.

I wish to express my warmest thanks to all my colleagues and friends at Linköping University, especially at the Division of Communication Systems. Precious was our enthusiastic discussions and collaborations in the group seminars, Fika chats, and informal meetings. It was an unforgettable experience that will leave marks beyond this thesis on me. I would also like to thank the teaching mentors for dedicating their time to enrich my teaching skills.

I would take this opportunity to gratefully acknowledge the "5Gwireless" project (H2020 Marie Skłodowska-Curie Innovative Training Networks), ELLIIT, and Ericsson's Research Foundation, for their financial support of the research conducted during my Ph.D. studies.

From the first day of my Ph.D. studies, I had the privilege to share an office with Chien, my ex-officemate, co-author, and friend, for all his support, fruitful discussions, and inspiration.

I always remain grateful to Arash, who is a brother to me. It has been almost two decades. Knowing that he is always there to have my back makes me stay strong.

Living in three continents and four different countries sometimes challenged 
me very hard in life. Yet, I am delighted as I have got many friends around the globe. To all the true friends that I have had the privilege to meet during this journey, specifically: Alyson, Hossein Doroud, Mahmoud Delvar, Alok, Alireza Haghighatkhah, Ahmed Ibrahim, Mohammed S. Elbamby, Giovanni, Silvia, Ema, Özgecan, Vedat, Kamil, Deniz, Marcus, Ashkan, Shahriar, Parisa, Pedram and Nader you helped me to see other important things in life. Without your closeness and friendship, I do not even want to imagine how this journey could have been. Particularly, I was blessed to have Kamiar and Parinaz as my friends and family in Sweden. Thank you for all the support.

Nastaran, I am speechless to find words to thank you. You are the meaning of life and the most important person to me. Your unconditional love and support keep me moving, and never give up.

Last but not least, I am incredibly grateful to my parents and Arezoo, my lovely sister, for their unconditional love, prayers, support, and care. I would also thank Nastaran's family for their inspiration, support, and positive energy.

Amin Ghazanfari Linköping, June 2021 


\section{List of Abbreviations}

$5 \mathrm{G}$

fifth generation of cellular network technology

AoA

angle of arrival

AWGN

additive white Gaussian noise

BS

base station

CDF

cumulative distribution function

CSI

channel state information

$\mathrm{CU}$

cellular user

D2D

device-to-device

$\mathrm{DL}$

downlink

DNN

deep neural network

EE

energy efficiency

FDD

frequency-division duplex

GM

geometric mean

i.i.d.

independent and identically distributed

LoS

line-of-sight

MIMO

multiple-input multiple-output

MMF

max-min fairness

MMSE

minimum mean-square error

mMTC

massive machine-type communication

MR

maximum ratio

NLoS

non line-of-sight

NMSE

normalized mean square error

NR

New Radio

NW-MMF

network-wide max-min fairness 


$\begin{array}{ll}\text { NW-PF } & \text { network-wide proportional fairness } \\ \text { PC } & \text { power control } \\ \text { QoS } & \text { quality-of-service } \\ \text { RF } & \text { radio frequency } \\ \text { SE } & \text { spectral efficiency } \\ \text { SISO } & \text { single-input single-output } \\ \text { SNR } & \text { signal-to-noise ratio } \\ \text { SINR } & \text { signal-to-interference-plus-noise ratio } \\ \text { TDD } & \text { time-division duplex } \\ \text { UL } & \text { uplink } \\ \text { URLLC } & \text { ultra-reliable low-latency communication } \\ \text { UatF } & \text { use-and-then-forget } \\ \text { ZF } & \text { zero-forcing }\end{array}$




\section{Chapter 1}

\section{Introduction}

\subsection{Motivation}

The idea of cellular network is more than half a century old and dates back to 1947 [1]. The idea was supported by the first practical implementation in 1979 by Nippon Telegraph and Telephone (NTT). Before the cellular network technology, the wireless transmitter usually communicated directly with the receiver, even if they were located very far apart. The core intention of using cellular network technology is to overcome two main limitations of early wireless systems [2,3]:

1. High attenuation of signals when transmitted over a considerable distance. This severely limits wireless communication performance over a wide coverage area and requires very high transmit power.

2. Significant interference will occur if other transmissions occur (at the same time and frequency) in the area between the transmitter and receiver. To avoid that, only one transmission was permitted, which led to few transmissions taking place simultaneously in a country [4].

Cellular communication is a fundamental technology where the transmitter and receiver communicate with nearby base stations (BSs) instead of directly with each other. This type of communication leads to wireless networks where transmissions take place over shorter distances and thereby more efficient utilization of the limited available frequency band for wireless communication [3]. Figure 1 shows a cellular network in which the coverage area is divided into multiple cells, where each cell has a fixed BS that the devices in the cell are connected to, and the BS provides service for them. To further enhance the frequency band's utilization, the frequency band is divided into the frequency sub-bands, and each cell uses some of the subbands. Cellular system also considers reusing the frequency sub-bands between 


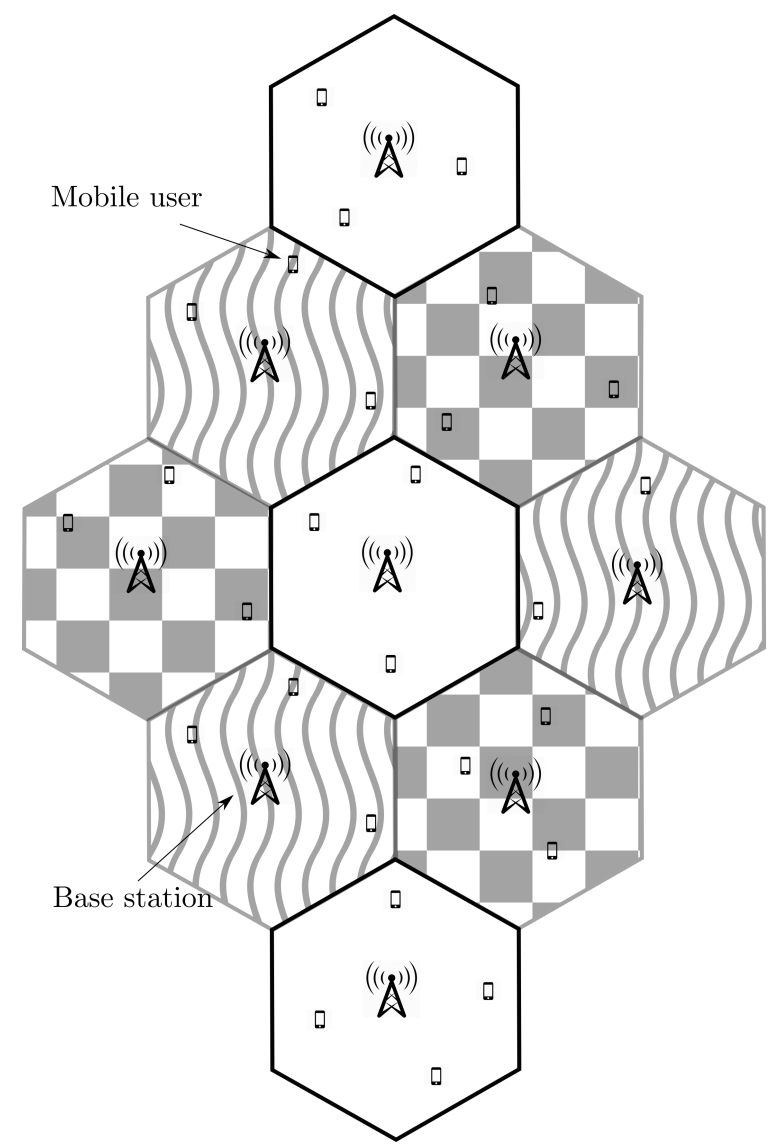

Figure 1: A basic cellular network. The same pattern corresponds to utilization of the same frequency sub-bands.

the cells if sufficient distances separate them, which is called frequency reuse. Therefore, the frequency reuse is selected to balance intercell interference and frequent reuse of the frequency bands. Note that in Figure 1, the cells with the same pattern use the same frequency sub-bands. Hence, cellular networks can provide service for a larger number of users in a given area and thereby accommodated the widespread usage of wireless communication. Ultimately, cellular technology became dominant in the commercial wireless communication systems [4] across the world.

Since 1979, cellular communication systems have developed significantly and became a revolutionary technology that is well utilized for the daily life of humans worldwide. Cellular networks were initially developed for providing voice commu- 
nication services to mobile users. However, the advancement of mobile devices and wireless technologies enabled mobile users to also benefit from data transmission of various kinds. These advancements facilitate data-hungry applications and services such as online gaming and video calls by mobile users. In the past few decades, this trend caused an ever-increasing demand for higher data rates and more data traffic [5]. The development of new communication technologies such as massive machine-type communication (mMTC) and ultra-reliable low-latency communication (URLLC) also play an essential role in the increase of data traffic [6]. Cisco has predicted that a similar growth will occur in the next decade [7], and Ericsson has reported annual growth of 28 percent between 2020 and 2026 [8]. Industrial and research parties have made a notable joint effort to deal with the ever-increasing inclination for higher data traffic resulting in gradual improvements of the wireless networks technologies. Different generations of wireless communication systems were released approximately every 10 years. $5 \mathrm{G}$ which is also known as new radio (NR) [9], is the latest generation of wireless communication that is implemented in different countries around the world. Each new generation is based on a standard that continues to be evolved even when a new generation is released [5].

The following three ways are the leading solutions proposed to handle the higher demand for data traffic in the wireless networks [10]:

1. Allocating more frequency bands and higher bandwidths.

2. Densification of networks by deploying more BSs in the coverage areas [11-13].

3. Improving the spectral efficiency (SE) per cell.

Note that the SE is defined as the amount of information transferred per second over one $\mathrm{Hz}$ of bandwidth. This thesis is primarily concerned with improving the SE.

\subsubsection{Massive MIMO}

This subsection introduces Massive MIMO. That is one of the influential technologies that significantly enhance the SE of future cellular networks. Massive MIMO was first introduced in [14] and it is considered as a backbone technology of 5G networks $[5,15,16]$. In Massive MIMO systems, each BS is implemented with hundreds of antenna elements, and each BS is assisting tens of single-antenna users [17, 18]. The Massive MIMO BSs serve the users over the same time-frequency block [19]. This stands in contrast to conventional BSs that use one or a few antennas and usually only serve one user per time-frequency block. Here, we list some of the 
main benefits of having a large number of antennas at the BSs in Massive MIMO systems $[10,20]$.

1. It enhances the SE per cell as it provides simultaneous data transmission to more users. The interference between users is dealt with using directional transmissions.

2. By providing narrow directional transmission and reception of signals, it increases the received signal power, and consequently for a given data rate, it requires lower transmit power. Hence, it also increases the energy efficiency (EE) of the network.

A detailed explanation of the benefits of Massive MIMO is provided in Chapter 2 .

\subsubsection{D2D Communication}

D2D communication was introduced as a smart paradigm to enhance the limited bandwidth utilization in cellular networks [21]. In D2D communication, instead of sending the data through an intermediate point, i.e., the BS in the cellular system, D2D offers direct communication between users [22]. Unlike pre-cellular wireless communication, this feature is only utilized when it is beneficial compared to cellular communication. To be more precise, D2D communication is practical in the case of short distances between transmitter and receiver [22,23]. D2D communication can either get its dedicated resources for communication or share the same resources as cellular users. The case of sharing the same resource as cellular users is known as D2D underlay communication [24], which is considered in this thesis. It can potentially improve the SE of cellular networks as we can serve more users in a given area for a given resource compared to conventional cellular networks. It also enables low power data transmission (higher EE and longer battery life of cellular devices) $[25,26]$.

\subsubsection{Topic and Motivation of the Thesis}

The primary motivation of this thesis is to investigate some possible approaches to increase the SE of wireless cellular networks. To be more specific, we mainly focus on Massive MIMO and D2D underlay communication as two possible technologies to enhance the SE of cellular networks. For example, in the first part, the motivation is to couple the benefits mentioned above for D2D underlay communication within Massive MIMO frameworks to intensify the increase in SE of cellular networks. In this part, we aim at improving the SE of a D2D underlaid multi-cell Massive MIMO network by utilizing different power control schemes. The second part 
of the thesis investigates the scalability issue of some well-known power control schemes for multi-cell Massive MIMO frameworks and provides a scalable solution for multi-cell Massive MIMO to enhance SE of such networks. In the last part of the thesis, we focus on studying downlink channel estimation for multi-cell Massive MIMO networks. The goal is to provide solutions for the limited performance of typical downlink channel estimation schemes for multi-cell Massive MIMO networks under certain channel conditions. These new approaches will help to improve SE performance of multi-cell Massive MIMO networks. A more detailed explanation of D2D communication and Massive MIMO technology is given in later parts of the thesis.

\subsection{Contributions of the Thesis}

This thesis focuses on a couple of practical aspects to improve SE of multi-cell Massive MIMO networks. It consists of an introductory part and a collection of research papers. The introduction part covers a brief explanation of Massive MIMO, power control, optimization approaches, and neural networks.

The second part consists of research papers in which the first two articles focus on explaining and evaluating the practical use cases of power control in multi-cell Massive MIMO networks, with and without D2D communications. More specifically, in Paper A we investigate power control for D2D underlaid Massive MIMO networks. As our second contribution, Paper B, proposes a new scalable power control framework that solves scalability issues of two well-known classical fairness power control approaches in a multi-cell Massive MIMO network. In Paper C, the last publication in the second part of the thesis, the focus is to study downlink channel estimation in a multi-cell Massive MIMO network. Paper C proposes one model-based and one data-driven approach for downlink channel estimation in multi-cell Massive MIMO networks. The next section contains the publication information and abstracts of the papers mentioned above. It also consists of a list of other publications excluded from the thesis as they are the preliminary conference versions of corresponding journal articles. 


\subsection{Papers Included in the Thesis}

\section{Paper A: Optimized Power Control for Massive MIMO with Underlaid D2D Communications}

Authored by: Amin Ghazanfari, Emil Björnson, and Erik G. Larsson.

Published in the IEEE Transactions on Communications, volume 67, issue 4, pp. 2763-2778, December 2018.

Abstract: In this work, we consider device-to-device (D2D) communication that is underlaid in a multi-cell Massive multiple-input multiple-output (MIMO) system and propose a new framework for power control and pilot allocation. In this scheme, the cellular users (CU)s in each cell get orthogonal pilots which are reused with reuse factor one across cells, while all the D2D pairs share another set of orthogonal pilots. We derive a closed-form capacity lower bound for the CUs with different receive processing schemes. In addition, we derive a capacity lower bound for the D2D receivers and a closed-form approximation of it. We provide power control algorithms to maximize the minimum spectral efficiency (SE) and maximize the product of the SINRs in the network. Different from prior works, in our proposed power control schemes, we consider joint pilot and data transmission optimization. Finally, we provide a numerical evaluation where we compare our proposed power control schemes with the maximum transmit power case and the case of conventional multi-cell Massive MIMO without D2D communication. Based on the provided results, we conclude that our proposed scheme increases the sum SE of multi-cell Massive MIMO networks.

\section{Paper B: Enhanced Fairness and Scalability of Power Control Schemes in Multi-cell Massive MIMO}

Authored by: Amin Ghazanfari, Hei Victor Cheng, Emil Björnson, and Erik G. Larsson

Published in the IEEE Transactions on Communications, volume 68, issue 5, pp. 2878 - 2890, May 2020.

Abstract: This paper studies the transmit power optimization in multi-cell Massive multiple-input multiple-output (MIMO) systems. Network-wide max-min fairness (NW-MMF) and network-wide proportional fairness (NW-PF) are two well-known power control schemes in the literature. The NW-MMF focus on maximizing the fairness among users at the cost of penalizing users with good channel conditions. On the other hand, the NW-PF focuses on maximizing the sum spectral efficiency 
(SE), thereby ignoring fairness, but gives some extra attention to the weakest users. However, both of these schemes suffer from a scalability issue which means that for large networks, it is highly probable that one user has a very poor channel condition, pushing the SE of all users towards zero. To overcome the scalability issue of NW-MMF and NW-PF, we propose a novel power control scheme that is provably scalable. This scheme maximizes the geometric mean (GM) of the per-cell max-min SE. To solve this new optimization problem, we prove that it can be rewritten in a convex optimization form and then solved using standard tools. The simulation results highlight the benefits of our model which is balancing between NW-PF and NW-MMF.

\section{Paper C: Model-based and Data-driven Approaches for Downlink Massive MIMO Channel Estimation}

Authored by: Amin Ghazanfari, Trinh Van Chien, Emil Björnson, and Erik G. Larsson

Submitted to the IEEE Transactions on Communications.

Abstract: We study downlink channel estimation in a multi-cell Massive multipleinput multiple-output (MIMO) system operating in time-division duplex. The users must know their effective channel gains to decode their received downlink data. Previous works have used the mean value as the estimate, motivated by channel hardening. However, this is associated with a performance loss in non-isotropic scattering environments. We propose two novel estimation methods that can be applied without downlink pilots. The first method is model-based and asymptotic arguments are utilized to identify a connection between the effective channel gain and the average received power during a coherence block. This second method is data-driven and trains a neural network to identify a mapping between the available information and the effective channel gain. Both methods can be utilized for any channel distribution and precoding. For the model-aided method, we derive closed-form expressions when using maximum ratio or zero-forcing precoding. We compare the proposed methods with the state-of-the-art using the normalized mean-squared error and spectral efficiency (SE). The results suggest that the two proposed methods provide better SE than the state-of-the-art when there is a low level of channel hardening, while the performance difference is relatively small with the uncorrelated channel model. 


\subsection{Papers Not Included in the Thesis}

The following publications also contain the works that are done by the author but are excluded from the thesis since they are the preliminary versions of the following included papers: Paper A and Paper B.

\section{Power Control for D2D Underlay in Multi-cell Massive MIMO Networks}

Authored by Amin Ghazanfari, Emil Björnson, and Erik G. Larsson

Published in Proceedings of 22nd International ITG Workshop on Smart Antennas (WSA), pp. 1-8, March 2018.

This paper contains preliminary results of Paper A

Abstract: This paper proposes a new power control and pilot allocation scheme for device-to-device (D2D) communication underlaying a multi-cell Massive MIMO system. In this scheme, the cellular users in each cell get orthogonal pilots which are reused with reuse factor one across cells, while the D2D pairs share another set of orthogonal pilots. We derive a closed-form capacity lower bound for the cellular users with different receive processing schemes. In addition, we derive a capacity lower bound for the D2D receivers and a closed-form approximation of it. Then we provide a power control algorithm that maximizes the minimum spectral efficiency (SE) of the users in the network. Finally, we provide a numerical evaluation where we compare our proposed power control algorithm with the maximum transmit power case and the case of conventional multi-cell Massive MIMO without D2D communication. Based on the provided results, we conclude that our proposed scheme increases the sum spectral efficiency of multi-cell Massive MIMO networks.

\section{A Fair and Scalable Power Control Scheme in Multi-cell Massive MIMO} Authored by Amin Ghazanfari, Hei Victor Cheng, Emil Björnson, and Erik G. Larsson Published in Proceedings of IEEE International Conference on Acoustics, Speech and Signal Processing (ICASSP), pp.4499-4503, May 2019.

This paper contains preliminary results of Paper B.

Abstract: This paper studies the transmit power optimization in a multi-cell Massive multiple-input multiple-output (MIMO) system. To overcome the scalability issue of network-wide max-min fairness (NW-MMF), we propose a novel power control (PC) scheme. This scheme maximizes the geometric mean (GM) of the per-cell max-min spectral efficiency (SE). To solve this new optimization problem, 
we prove that it can be rewritten in a convex form and then solved using standard tools. To provide a fair comparison with the available utility functions in the literature, we solve the network-wide proportional fairness (NW-PF) PC as well. The NW-PF focuses on maximizing the sum SE, thereby ignoring fairness, but gives some extra attention to the weakest users. The simulation results highlight the benefits of our model which is balancing between NW-PF and NW-MMF. 



\section{Chapter 2}

\section{Massive MIMO}

This chapter is dedicated to briefly explaining some of the fundamental concepts in Massive MIMO technology. To be more specific, we first provide a background on Massive MIMO and highlight its main difference with conventional multi-user MIMO. Next, we explain some of the key properties of Massive MIMO technology. The main intention is to walk through the concepts used in the included research papers in this thesis. A more comprehensive description of Massive MIMO technology can be found in [10,27]. Finally, we provide a system model for multi-cell Massive MIMO networks and briefly explain channel estimation and data transmission for both uplink and downlink directions in the multi-cell Massive MIMO networks.

\subsection{Background}

Massive MIMO originated as an extension of a multi-user MIMO $[28,29]$ system operating in time-division duplex (TDD). A multi-user MIMO system is a system that is serving multiple users simultaneously by a multi-antenna BS [30,31]. Multiple antennas at the BS provide an array gain if adequately used. That means the BS performs directional beamforming towards the desired receiver which amplifies the received signal power. Moreover, the multiple antennas can be used for spatial multiplexing, which is the simultaneous transmission of multiple signals with different directional beamforming for serving multiple users at the same time and frequency. Spatial multiplexing can increase the SE per cell proportionally to the number of multiplexed users [32]. However, due to the limited number of antennas at the BSs in conventional multi-user MIMO, the system is only capable of simultaneous multiplexing of a small number of users.

The idea of Massive MIMO is initiated in the seminal paper of Marzetta [14]. 
Massive MIMO refers to a system that consists of BSs with hundreds of antennas that can serve tens of users simultaneously. The BSs serve all users over the same time-frequency block by spatial multiplexing [19,33]. Each antenna at a BS has a dedicated radio frequency $(\mathrm{RF})$ chain. Hence, we can benefit from fully digital beamforming in the network. Here, we highlight two offered benefits of Massive MIMO systems.

First, it enhances the SE per cell compared to the conventional MIMO systems as a result of spatial multiplexing more users with Massive MIMO BSs [27]. Furthermore, a large number of antennas at the Massive MIMO BSs increases the array gain, and the increased directivity reduces the inter-user interference, which results in higher SE per user. Therefore, Massive MIMO offers a significant boost in the per-cell SE of the cellular network.

Second, many antennas at the Massive MIMO BSs make the users' channels almost spatially orthogonal. This is one of the key properties of Massive MIMO, which is also known as favorable propagation $[10,27,34,35]$. As a result of favorable propagation, relatively simple processing schemes that treat interference as noise can be used efficiently. Due to a large number of antennas at the Massive MIMO BSs, the effect of small-scale fading of wireless propagation channels is averaged out. This phenomenon is known as channel hardening [10,27,36,37]. One can say that under channel hardening, the channels are more deterministic. Hence, we can perform resource allocation, power control, etc., over the large-scale fading only without having small-scale fading involved in the allocation or control process. Performing resource allocation over the large-scale fading reduces the complexity of signal processing in the system. Favorable propagation and channel hardening are further discussed in the next section.

Massive MIMO is an established technology, and it became part of the 5G NR standard. The benefits of Massive MIMO are proven analytically by the research community, and a significant effort has been made to prove the underlaying potential of Massive MIMO [38-40]. Later, it was supported with practical implementation and testbeds from both academia and industry [41,42]. Even though we have seen that Massive MIMO is a powerful technology with outstanding benefits, more research should be done to explore the true potentials of it. This thesis is dedicated to contribute in enhancing the potential benefits of Massive MIMO technology.

\subsection{Key Properties of Massive MIMO}

In this section, we discuss some essential aspects of Massive MIMO in further detail. In particular, we explain and define favorable propagation and channel hardening in a mathematical way. In addition, we present a brief discussion on the possible 
duplexing protocols for Massive MIMO systems and explain why one is preferred.

\subsubsection{Favorable Propagation}

Favorable propagation is a phenomenon that appears when the propagation channels of two cellular users are mutually orthogonal. Favorable propagation helps the BS to cancel co-channel interference between users without having to design advanced algorithms for interference suppression. Consequently, it enhances the SE of both users. Let us assume that we have a single cell consisting of a BS that has $M$ antennas and two single-antenna users. The vectors $\mathbf{g}_{1} \sim C \mathcal{N}\left(\mathbf{0}, \mathbf{I}_{M}\right)$ and $\mathrm{g}_{2} \sim C \mathcal{N}\left(\mathbf{0}, \mathbf{I}_{M}\right)$ denote the channel responses of the two users over a narrowband channel. These vectors are circularly symmetric complex Gaussian distributed with zero mean and correlation matrix $\mathbf{I}_{M}$ and this channel model is known as independent and identically distributed (i.i.d) Rayleigh fading. In case the channel vectors are orthogonal, the inner product satisfies [34]

$$
\mathbf{g}_{1}^{\mathrm{H}} \mathbf{g}_{2}=0 \text {. }
$$

The BS can then separate the received signal from these two users without any loss in the desired signals. Let us assume $x_{1}$ and $x_{2}$ denote the data signals transmitted by these two users. The received signal at the BS is given by

$$
\mathbf{y}=\mathrm{g}_{1} x_{1}+\mathrm{g}_{2} x_{2} .
$$

Assuming the BS has perfect knowledge of both channel vectors, it can cancel the interference between the users by taking the inner product of the received signal $y$ with the channel of the desired user. In addition, the noise effect is neglected for simplicity. For example, when considering user 1, the inner product is

$$
\mathbf{g}_{1}^{\mathrm{H}} \mathbf{y}=\left\|\mathbf{g}_{1}\right\|^{2} x_{1}+\mathbf{g}_{1}^{\mathrm{H}} \mathbf{g}_{2} x_{2}=\left\|\mathbf{g}_{1}\right\|^{2} x_{1},
$$

that gives the desired signal of user one, since the part $\mathbf{g}_{1}^{\mathrm{H}} \mathbf{g}_{2} x_{2}$ is zero thanks to orthogonality of the vectors in (1). This is an ideal situation for the BS, which is why it is called favorable propagation; however, this is not very likely to occur in practice or if the channel vectors are drawn from random distributions. However, in the case of Massive MIMO BSs, we can show that an approximate favorable propagation can happen asymptotically in the case of Rayleigh fading channels [27]. It is defined as the inner product of the two normalized vectors satisfying [34]:

$$
\frac{\mathbf{g}_{1}^{\mathrm{H}} \mathbf{g}_{2}}{M} \rightarrow 0,
$$

with almost sure convergence when $M \rightarrow \infty$, therefore as the number of antenna $M$ grows large, these two channel vectors are asymptotically orthogonal. We denote that the above property holds for Rayleigh fading channels. 


\subsubsection{Channel Hardening}

In this part, we define and explain the concept of channel hardening. Channel hardening refers to the fact that the channel is less susceptible to the small-scale fading effects and behaves more like a deterministic channel when utilizing all the antennas [43]. Let us assume $\mathbf{g} \sim \mathcal{C N}\left(\mathbf{0}, \mathbf{I}_{M}\right)$ is the channel vector of an arbitrary user towards a Massive MIMO BS with $M$ antennas, asymptotic channel hardening is defined as [10]

$$
\frac{\|\mathbf{g}\|^{2}}{\mathbb{E}\left\{\|\mathbf{g}\|^{2}\right\}} \rightarrow 1,
$$

when $M \rightarrow \infty$ the convergence holds almost surely. Note that a squared norm of the kind in (5) appeared in (3) when the BS processed the received signal, which is why its value is important for determining the communication performance. Asymptotic channel hardening implies that the value of $\|\mathrm{g}\|^{2}$ is close to its mean value, so the variations are small. This phenomenon is an extension of the spatial diversity concept from conventional small-scale MIMO systems to the case of having a large number of antennas at the BSs. Channel hardening implies that the channel quality $\|\mathbf{g}\|^{2}$ for a given channel realization is well approximated by the average channel quality $\mathbb{E}\left\{\|\boldsymbol{g}\|^{2}\right\}$. Hence, if we want to select power coefficients based on the channel quality, we do not need to adapt them to the small-scale fading variations, but the same power can be used for a long time period. We consider channel hardening as one of the essential benefits of Massive MIMO systems, which helps us to propose practical power control schemes in the included papers in this thesis.

\subsubsection{Duplexing Protocol}

This subsection briefly explains the possible duplexing protocols for Massive MIMO systems, i.e., TDD and frequency-division duplex (FDD). In order to process the uplink and downlink signals, each BS needs to estimate the channel vectors of its serving users in each channel coherence block. A coherence block is defined as the time-frequency block in which the fading channel is static. In Massive MIMO, we assume that full statistical channel state information is available at the BSs. However, one should perform channel estimation at each BS, to obtain the instantaneous channel state information. Channel estimation is performed via pilot transmission [10,27].

In the pilot transmission phase, each transmitter (e.g., a cellular user in uplink pilot transmission) sends one of the sequences from the set of predefined pilot signal sequences known by both the transmitter and receiver (e.g., the BS in the 
uplink pilot transmission). To estimate the channel from the transmitter, the receiver compares the signal with the "true" signal from the set. To support the pilot transmission of multiple transmitters in Massive MIMO systems, we generally prefer to have the same number of orthogonal pilot sequences as the number of transmitting antennas. It is also desirable to keep the pilot signals as short as possible to use most of the resources in a coherence block for data transmission. Pilot transmission for channel estimation in downlink and uplink of a Massive MIMO system requires a different number of pilot symbols. In the uplink, assuming that we have $K$ single-antenna users, the system requires $K$ pilot signal sequences to estimate the uplink channels. However, if the BS has $M$ antennas, pilot transmission in the downlink requires $M$ pilot signals, where $M \gg K$ is normal in Massive MIMO systems.

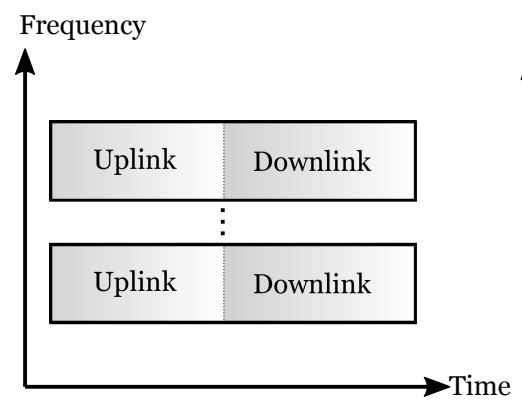

(a) TDD Protocol

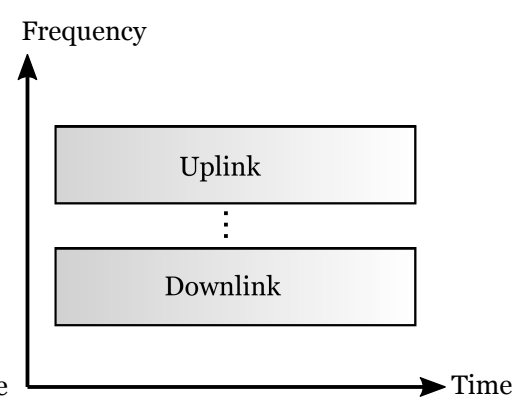

(b) FDD Protocol

Figure 2: Time-frequency separation of coherence block for TDD and FDD protocol [10].

TDD refers to separating the uplink and downlink transmissions in the timedomain while using the whole bandwidth; assuming that both happen in the same coherence time, channel reciprocity holds. It means that the channel is the same in both directions. Hence, by doing channel estimation in one direction (i.e., uplink here), the estimated channel is valid for the other direction (i.e., downlink) as well. Therefore, in TDD Massive MIMO systems, we require $K$ pilot sequences only. Hence, channel estimation does not depend on $M$.

In FDD, the uplink and downlink transmission occur simultaneously but in different frequency bands. Hence, due to the different frequency bands for uplink and downlink, the channel reciprocity does not hold [10]. Consequently, we need to estimate the channels separately for each direction. Therefore, we require both uplink and downlink pilots for channel estimation in FDD. In the downlink, we need $M$ pilot signal sequences and an additional $M$ signals for reporting back the estimated channel to the BS in the uplink. Besides, we need $K$ pilots for uplink channel estimation. In total, assuming the resources are equally decided between 
uplink and downlink, FDD needs $(2 M+K) / 2$ pilot signals. The time-frequency separation of these two protocols is illustrated in Figure 2, please note that in FDD there is usually many $100 \mathrm{MHz}$ between the uplink and downlink.

One can see that channel estimation overhead in TDD Massive MIMO is substantially smaller than in FDD Massive MIMO, and it is not scaled with $M$. Therefore, TDD is a preferable duplexing mode for Massive MIMO systems, and we assume TDD for the included papers in this thesis.

\subsection{System Model}

This section describes a system model for a multi-cell Massive MIMO network, which we use to explain concepts such as channel estimation, data transmissions, and power control approaches in the thesis. We consider a multi-cell Massive MIMO network consisting of $L$ cells, and each cell has one BS. The number of antennas at the BSs are equal, and each BS has $M$ antennas. Besides, each cell serves $K$ single antenna users in the coverage area. Figure 3 illustrates the multi-cell Massive MIMO model.

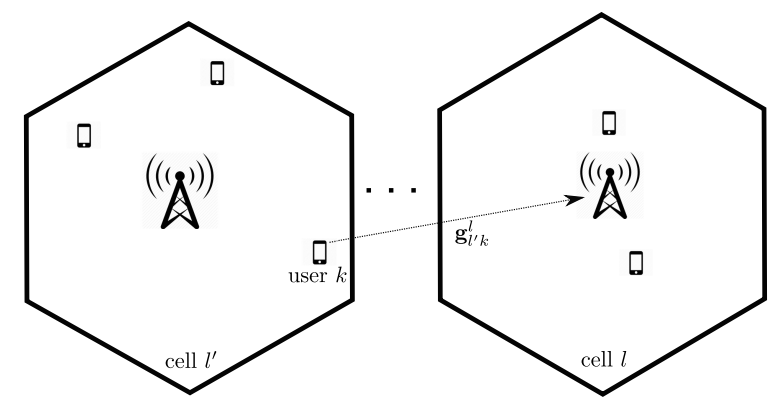

Figure 3: Illustration of multi-cell setup.

In this system model, we assume that the system operates in TDD mode. We use a block fading assumption to model wireless propagation channels that are varying over time and frequency. This is a time-frequency block of $\tau_{c}$ samples in which the channel is constant and frequency-flat, which is defined as the coherence block of the channel. A stationary ergodic random process is used to model that the channel is changing independently over different blocks. The size of $\tau_{c}=T_{c} B_{c}$ which depends on the coherence time and bandwidth denoted by $T_{c}$ and $B_{c}$, respectively [27, Ch. 2], [10, Ch. 2]. We denote the channel response between user $k$ located in cell $l^{\prime}$ towards the BS in cell $l$ by the vector $\mathbf{g}_{l^{\prime} k}^{l} \sim C \mathcal{N}\left(\mathbf{0}, \beta_{l^{\prime} k}^{l} \mathbf{I}_{M}\right)$ which is depicted by the dashed arrow in the Figure 3. This channel model is called uncorrelated Rayleigh fading [10]. The channels take one independent realization 
in each coherence block. The nonnegative $\beta_{l^{\prime} k}^{l}$ is the large-scale fading coefficient for this channel response.

\subsection{Channel Estimation}

In this setup, we assume that the BSs do not have channel state information (CSI) a priori. Since the channels are changing over each coherence block, the system requires to carry out channel estimation in each coherence block. Therefore, $\tau_{p} \geq K$ symbols are dedicated to uplink pilot transmission from the users to the $\mathrm{BSs}$, which gives room for transmitting deterministic pilot sequences of length $\tau_{p}$. Furthermore, we use the remaining $\tau_{c}-\tau_{p}$ symbols for uplink and downlink data transmission. Figure 4 illustrates one coherence block of TDD Massive MIMO.

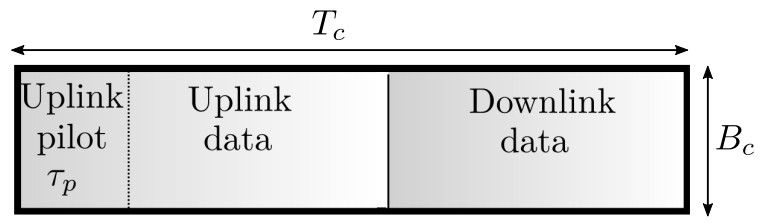

Figure 4: One coherence block in a TDD Massive MIMO system.

As we discussed in Subsection 2.2.3, due to channel reciprocity, the estimated channel for the uplink can be used for the downlink direction as well. The $K$ users in a cell are assumed to be using orthogonal pilot sequences from the $\tau_{p}$ samples. However, as we have a limited number of pilot sequences in the network ( $L K$ is generally more prominent than $\tau_{p}$ ), we need to reuse each pilot sequence in multiple cells according to some pilot reuse policy.

Reusing pilot sequences will cause mutual interference in the pilot transmission of the users using the same pilot sequence. This mutual interference is called pilot contamination in the literature. Consequently, pilot contamination affects the channel estimation quality and is one of the limiting factors of Massive MIMO systems. During the pilot transmission in the considered system model, the received pilot signal at $\mathrm{BS} l$ is denoted as $\mathrm{Y}_{l}^{\mathrm{p}} \in \mathbb{C}^{M \times \tau_{p}}$

$$
\mathbf{Y}_{l}^{\mathrm{p}}=\sum_{l^{\prime}=1}^{L} \sum_{k=1}^{K} \sqrt{\tau_{p} \rho_{\mathrm{ul}}} \mathbf{g}_{l^{\prime} k}^{l}\left(\boldsymbol{\phi}_{l^{\prime} k}\right)^{\mathrm{H}}+\mathbf{W}_{l},
$$

where $\rho_{\mathrm{ul}}$ is the maximum uplink transmit power and $\phi_{l k} \in \mathbb{C}^{\tau_{p}}$ indicates the orthonormal pilot sequence assigned for user $k$ in cell $l$ and $\mathbf{W}_{l} \in \mathbb{C}^{M \times \tau_{p}}$ is the normalized additive white Gaussian noise at the BS $l$ that consists of independent entries which are having the distribution $C \mathcal{N}(0,1)$. Note that, all users 
use full transmit power for pilot transmission, therefore there are no power control coefficients in (6). We denote the pilot matrix used by the users in cell $l$ as $\Phi_{l}=\left[\phi_{l 1}, \ldots, \phi_{l K}\right]$. Each BS multiplies the received signal matrix during pilot transmission with its pilot matrix to despread the signals. Therefore, the received pilot signal at BS $l$ is, after despreading by the pilot matrix $\Phi_{l}$, given by

$$
\mathrm{Y}_{l}^{\mathrm{p}} \boldsymbol{\Phi}_{l}=\sum_{l^{\prime}=1}^{L} \sum_{k=1}^{K} \sqrt{\tau_{p} \rho_{\mathrm{ul}}} \mathbf{g}_{l^{\prime} k}^{l}\left(\phi_{l^{\prime} k}\right)^{\mathrm{H}} \boldsymbol{\Phi}_{l}+\mathbf{W}_{l} \boldsymbol{\Phi}_{l} .
$$

The minimum mean square error (MMSE) estimates of $\mathbf{g}_{l^{\prime} k}^{l}$ is denoted as $\hat{\mathbf{g}}_{l^{\prime} k}^{l} \sim \mathcal{C N}\left(0, \gamma_{l^{\prime} k}^{l} \mathbf{I}_{M}\right)$ that follows the standard MMSE estimation approach in the literature, e.g., $[10,27,44]$ given by

$$
\hat{\mathbf{g}}_{l^{\prime} k}^{l}=\frac{\sqrt{\tau_{p} \rho_{\mathrm{ul}}} \beta_{l^{\prime} k}^{l}}{1+\tau_{p} \rho_{\mathrm{ul}} \sum_{l^{\prime \prime} \in \mathcal{P}_{l}} \beta_{l^{\prime \prime} k}^{l}} \mathbf{Y}_{l}^{\mathrm{p}} \boldsymbol{\phi}_{l k}, \quad l^{\prime} \in \mathcal{P}_{l},
$$

and the mean-square of the channel estimate $\hat{\mathbf{g}}_{l^{\prime} k}^{l}$ is

$$
\gamma_{l^{\prime} k}^{l}=\frac{\tau_{p} \rho_{\mathrm{ul}}\left(\beta_{l^{\prime} k}^{l}\right)^{2}}{1+\tau_{p} \rho_{\mathrm{ul}} \sum_{l^{\prime \prime} \in \mathcal{P}_{l}} \beta_{l^{\prime \prime} k}^{l}}, \quad l^{\prime} \in \mathcal{P}_{l},
$$

where $\mathcal{P}_{l}$ is the set of the BSs that are using the same $K$ pilot sequences as BS $l$. We also assume that for the BSs that are sharing the same set of pilot sequences, users with index $k$ utilize an identical pilot sequence for $k=1, \ldots, K$. Please note that even though we focus on the uncorrelated Rayleigh fading channel model in this introduction, we also considered correlated Rayleigh fading channel models in the included papers.

Furthermore, by exploiting the channel reciprocity in TDD Massive MIMO, the CSI obtained at the BSs is a legitimate estimate for the downlink channel. BSs use the obtained CSI knowledge to perform precoding in the downlink and combining in the uplink data communications. Hence, in the downlink, to apply precoding at the BSs, we can take the full benefit from channel reciprocity of TDD operation. Thanks to channel reciprocity in TDD, the amount of pilot resources required for TDD-based Massive MIMO is independent of the number of antennas at the BSs as discussed in Section 2.2.3.

As we considered in the current system model, using uncorrelated Rayleigh fading is a common assumption to model channels in Massive MIMO papers. Having many antennas per BS in a Massive MIMO setup and the assumption of 
having an uncorrelated Rayleigh fading model offers a high channel hardening level. The BSs use the MMSE estimate of uplink channels to transmit data toward desired users in the downlink. To coherently decode the transmitted data at the user side, each user should know the effective channel gain, i.e., product of the channel and precoding vectors that are varying over coherence blocks, instead of allocating downlink pilots to estimate the downlink channels at the users for decoding downlink data transmission which has extra pilot overhead. One common approach is to use the mean value of the effective channel gain at the users. This is a reasonable assumption, and the effective channel gain is relatively close to its mean value thanks to channel hardening. This approach has a relatively good performance compared to the downlink pilot approach without suffering from extra pilot overhead. However, if the channel hardening level is low, the performance of using the mean is decreasing. We investigate this problem in paper $\mathrm{C}$ and study some new approaches to solve this issue based on using downlink transmitted data to estimate the users' effective channel gains.

\subsection{Data Transmission}

The data transmission can occur in both uplink and downlink directions. In the uplink data transmission, cellular users transmit data towards their serving BS. The received signal during data transmission at BS $l$ is

$$
\mathbf{y}_{l}=\sum_{k=1}^{K} \sqrt{\rho_{\mathrm{ul}} \eta_{l k}} \mathbf{g}_{l k}^{l} s_{l k}+\sum_{\substack{l^{\prime}=1 \\ l^{\prime} \neq l}}^{L} \sum_{k=1}^{K} \sqrt{\rho_{\mathrm{ul}} \eta_{l^{\prime} k}} \mathbf{g}_{l^{\prime} k}^{l} s_{l^{\prime} k}+\mathbf{w}_{l},
$$

where $s_{l k}$ is the zero mean and unit variance data symbol transmitted from user $k$ in cell $l$ and $\eta_{l k} \in[0,1]$ denotes the power control coefficient of user $k$ located in cell $l$. It is the power control coefficients that will be optimized in this thesis. In addition, $\rho_{\mathrm{ul}}$ is the maximum uplink transmit power. $\mathbf{w}_{l} \sim C \mathcal{N}\left(\mathbf{0}, \mathbf{I}_{M}\right)$ indicates the normalized additive white Gaussian noise at BS $l$. The actual noise variance can be included in either the large-scale fading coefficients or the maximum transmit powers, without loss of generality. In order to decode the transmitted data signal from user $k$ in cell $l$, at the BS $l$, the BS multiplies the received signal with a receive combining vector $\mathbf{a}_{l k} \in \mathbb{C}^{M}$. The resulting received data signal of user $k$ in cell $l$ at 
the BS $l$ can be expressed as

$$
\begin{gathered}
\mathbf{a}_{l k}^{\mathrm{H}} \mathbf{y}_{l}=\sqrt{\rho_{\mathrm{ul}} \eta_{l k}} \mathbf{a}_{l k}^{\mathrm{H}} \mathbf{g}_{l k}^{l} s_{l k}+\sum_{\substack{k^{\prime}=1, k^{\prime} \neq k}}^{K} \sqrt{\rho_{\mathrm{ul}} \eta_{l k^{\prime}}} \mathbf{a}_{l k}^{\mathrm{H}} \mathbf{g}_{l k^{\prime}}^{l} s_{l k^{\prime}}+ \\
\sum_{\substack{l^{\prime}=1, k^{\prime}=1 \\
l^{\prime} \neq l}}^{B} \sqrt{\rho_{\mathrm{ul}} \eta_{l^{\prime} k^{\prime}}} \mathbf{a}_{l k}^{\mathrm{H}} \mathbf{g}_{l^{\prime} k^{\prime}}^{l} s_{l^{\prime} k^{\prime}}+\mathbf{a}_{l k}^{\mathrm{H}} \mathbf{w}_{l},
\end{gathered}
$$

where the first term is the desired part of the received signal, the second term is the intracell interference from other users in cell $l$, the third term is intercell interference coming from other users in the other cells. We rewrite the received data signal of user $k$ in cell $l$ by adding and subtracting $\sqrt{\rho_{\mathrm{ul}} \eta_{l k} \mathbb{E}}\left\{\mathbf{a}_{l k}^{\mathrm{H}} \mathbf{g}_{l k}^{l}\right\} s_{l k}$ term as

$$
\begin{aligned}
\mathbf{a}_{l k}^{\mathrm{H}} \mathbf{y}_{l} & =\sqrt{\rho_{\mathrm{ul}} \eta_{l k}} \mathbb{E}\left\{\mathbf{a}_{l k}^{\mathrm{H}} \mathbf{g}_{l k}^{l}\right\} s_{l k}+\sqrt{\rho_{\mathrm{ul}} \eta_{l k}}\left(\mathbf{a}_{l k}^{\mathrm{H}} \mathbf{g}_{l k}^{l}-\mathbb{E}\left\{\mathbf{a}_{l k}^{\mathrm{H}} \mathbf{g}_{l k}^{l}\right\}\right) s_{l k} \\
& +\sum_{\substack{k^{\prime}=1, k^{\prime} \neq k}}^{K} \sqrt{\rho_{\mathrm{ul}} \eta_{l k^{\prime}}} \mathbf{a}_{l k}^{\mathrm{H}} \mathbf{g}_{l k^{\prime}}^{l} s_{l k^{\prime}}+\sum_{\substack{l^{\prime}=1 \\
l^{\prime} \neq l}}^{L} \sum_{k^{\prime}=1}^{K} \sqrt{\rho_{\mathrm{ul}} \eta_{l^{\prime} k^{\prime}}} \mathbf{a}_{l k}^{\mathrm{H}} \mathbf{g}_{l^{\prime} k^{\prime}}^{l} s_{l^{\prime} k^{\prime}}+\mathbf{a}_{l k}^{l} \mathbf{w}_{l},
\end{aligned}
$$

where the first term is treated as the desired part of the received signal and the rest of the terms are treated as noise in the signal detection. We can then use the use-and-then-forget (UatF) technique [27, Ch. 3] to lower bound the capacity of each of the users, using the capacity bound for a deterministic channel with additive non-Gaussian noise provided in [27, Sec. 2.3]. Note that UatF reflect the fact that we utilize the channel estimates during the combining and then forget them before doing signal detection [10]. We calculate the lower bound on the capacity of user $k$ in cell $l$ as

$$
\mathrm{SE}_{l k}^{\mathrm{UL}}=\left(1-\frac{\tau_{p}}{\tau_{c}}\right) \log _{2}\left(1+\mathrm{SINR}_{l k}^{\mathrm{UL}}\right),
$$

where $\operatorname{SINR}_{l k}^{\mathrm{UL}}$ is the effective signal-to-interference-plus-noise ratio (SINR) of user $k$ in cell $l$ for uplink data transmission

$$
\operatorname{SINR}_{l k}^{\mathrm{UL}}=\frac{\rho_{\mathrm{ul}} \eta_{l k}\left|\mathbb{E}\left\{\mathbf{a}_{l k}^{\mathrm{H}} \mathbf{g}_{l k}^{l}\right\}\right|^{2}}{\sum_{l^{\prime}=1}^{L} \sum_{k^{\prime}=1}^{K} \rho_{\mathrm{ul}} \eta_{l^{\prime} k^{\prime}} \mathbb{E}\left\{\left|\mathbf{a}_{l k}^{\mathrm{H}} \mathbf{g}_{l^{\prime} k^{\prime}}^{l}\right|^{2}\right\}-\rho_{\mathrm{ul}} \eta_{l k}\left|\mathbb{E}\left\{\mathbf{a}_{l k}^{\mathrm{H}} \mathbf{g}_{l k}^{l}\right\}\right|^{2}+\mathbb{E}\left\{\left\|\mathbf{a}_{l k}\right\|^{2}\right\}} .
$$

Note that the expectations are with respect to the channel realizations. The provided bound works for any arbitrary combining vector $\mathbf{a}_{l k}$. However, in the 
discussion here and using in later chapters, we apply maximum ratio (MR) combining at each BS during the uplink data transmission phase. BS $l$ uses the channel estimates to detect the signal of its own user and for user $k$ the combining vector is defined as $\mathbf{a}_{l k}=\hat{\mathbf{g}}_{l k}^{l}$. By applying this combining vector, we can get the following closed form expression on the lower bound on the capacity of user $k$ in cell $l$ as

$$
\operatorname{SINR}_{l k}^{\mathrm{UL}}=\frac{M \rho_{\mathrm{ul}} \gamma_{l k}^{l} \eta_{l k}}{1+\rho_{\mathrm{ul}} \sum_{l^{\prime}=1}^{L} \sum_{k^{\prime}=1}^{K} \beta_{l^{\prime} k^{\prime}}^{l} \eta_{l^{\prime} k^{\prime}}+M \rho_{\mathrm{ul}} \sum_{l^{\prime} \in \mathcal{P}_{l} \backslash\{l\}} \gamma_{l^{\prime} k}^{l} \eta_{l^{\prime} k}}
$$

We denote the lower bound in (13) as the SE of user $k$ in cell $l$. Note that we only provide the closed form results for the case of MR for the uplink data transmission in this chapter. We will use the effective SINR expression in this section to explain and discuss network-wide max-min and proportional fairness power control schemes in multi-cell Massive MIMO systems.

In the downlink data transmission, BSs transmit data towards their serving users using linear precoding vectors. The transmitted signal when utilizing linear precoding at BS $l$ is

$$
\mathbf{x}_{l}=\sum_{k=1}^{K} \sqrt{\rho_{\mathrm{d} \mathbf{l}} \eta_{l k}} \mathbf{v}_{l k} s_{l k}
$$

where $s_{l k}$ is the zero mean and unit variance data symbol transmitted from $l$ th BS to its serving user $k$ and $\eta_{l k} \in[0,1]$ denotes the power control coefficient allocated for data transmission to user $k$ and $\rho_{\mathrm{dl}}$ is the maximum downlink transmit power of BS $l$. $\mathbf{v}_{l k} \in \mathbb{C}^{M}$ is the precoding vector for user $k$ in cell $l$. The received signal during data transmission at user $k$ in cell $l$ is

$$
\mathbf{y}_{l k}=\sum_{l^{\prime}=1}^{L}\left(\mathbf{g}_{l k}^{l^{\prime}}\right)^{\mathrm{H}} \mathbf{x}_{l^{\prime}}+w_{l k},
$$

and replacing $\mathbf{x}_{l^{\prime}}$ with (16), we have

$$
\begin{aligned}
\mathbf{y}_{l k} & =\sum_{l^{\prime}=1}^{L} \sum_{k^{\prime}=1}^{K} \sqrt{\rho_{\mathrm{d} \mathbf{l}} \eta_{l^{\prime} k^{\prime}}}\left(\mathbf{g}_{l k}^{l^{\prime}}\right)^{\mathrm{H}} \mathbf{v}_{l^{\prime} k^{\prime}} s_{l^{\prime} k^{\prime}}+w_{l k}, \\
= & \sqrt{\rho_{\mathrm{d} \mathbf{l}} \eta_{l k}}\left(\mathbf{g}_{l k}^{l}\right)^{\mathrm{H}} \mathbf{v}_{l k} s_{l k}+\sum_{\substack{k^{\prime}=1 \\
k^{\prime} \neq K}}^{K} \sqrt{\rho_{\mathrm{d} l} \eta_{l k^{\prime}}}\left(\mathbf{g}_{l k}^{l}\right)^{\mathrm{H}} \mathbf{v}_{l k^{\prime}} s_{l k^{\prime}} \\
& +\sum_{\substack{l^{\prime}=1, k^{\prime}=1 \\
l^{\prime} \neq l}}^{L} \sqrt{\rho_{\mathrm{dl}} \eta_{l^{\prime} k^{\prime}}} \mathbf{g}_{l k}^{l^{\prime}} \mathbf{v}_{l^{\prime} k^{\prime}} s_{l^{\prime} k^{\prime}}+w_{l k},
\end{aligned}
$$


where the first term is the desired part of the received signal for user $k$ in cell $l$, the second term is the intracell interference, i.e., coming from data transmission of BS $l$ to the other users in cell $l$, the third term is intercell interference coming from data transmission of other BS towards their serving users. $w_{l k} \sim C \mathcal{N}(0,1)$ indicates the normalized additive white Gaussian noise at user $k$ in cell $l$. To decode the desired signal, the common approach in Massive MIMO literature is that the users approximate $\left(\mathrm{g}_{l k}^{l}\right)^{\mathrm{H}} \mathbf{v}_{l k}$ with its statistical average value. Therefore, we can write the received signal at user $k$ in cell $l$ as

$$
\begin{aligned}
& \mathbf{y}_{l k}=\sqrt{\rho_{\mathrm{d} \mathbf{l}} \eta_{l k}} \mathbb{E}\left\{\left(\mathbf{g}_{l k}^{l}\right)^{\mathrm{H}} \mathbf{v}_{l k}\right\} s_{l k}+\sqrt{\rho_{\mathrm{d} \mathbf{l}} \eta_{l k}}\left(\left(\mathbf{g}_{l k}^{l}\right)^{\mathrm{H}} \mathbf{v}_{l k}-\mathbb{E}\left\{\left(\mathbf{g}_{l k}^{l}\right)^{\mathrm{H}} \mathbf{v}_{l k}\right\}\right) s_{l k} \\
& +\sum_{\substack{k^{\prime}=1 \\
k^{\prime} \neq k}}^{K} \sqrt{\rho_{\mathrm{d} l} \eta_{l k^{\prime}}}\left(\mathbf{g}_{l k}^{l}\right)^{\mathrm{H}} \mathbf{v}_{l k^{\prime}} s_{l k^{\prime}}+\sum_{\substack{l^{\prime}=1, k^{\prime}=1 \\
l^{\prime} \neq l}}^{L} \sqrt{\rho_{\mathrm{d} l} \eta_{l^{\prime} k}} \mathbf{g}_{l k}^{l^{\prime}} \mathbf{v}_{l^{\prime} k^{\prime}} s_{l^{\prime} k^{\prime}}+w_{l k},
\end{aligned}
$$

the first term indicates the desired signal over a deterministic average channel, the second term is the desired signal over an unknown channel, the third and fourth terms are intracell and intercell interference, respectively. Finally, one can use the received signal and the hardening bound $[10, \mathrm{Ch} .4]$ to get the lower bound on the capacity of user $k$ in cell $l$ as

$$
\mathrm{SE}_{l k}^{\mathrm{DL}}=\left(1-\frac{\tau_{p}}{\tau_{c}}\right) \log _{2}\left(1+\mathrm{SINR}_{l k}^{\mathrm{DL}}\right),
$$

where $\operatorname{SINR}_{l k}^{D L}$ is the effective SINR of user $k$ in cell $l$ for downlink data transmission

$$
\operatorname{SINR}_{l k}^{\mathrm{DL}}=\frac{\rho_{\mathrm{d} 1} \eta_{l k}\left|\mathbb{E}\left\{\left(\mathrm{g}_{l k}^{l}\right)^{\mathrm{H}} \mathbf{v}_{l k}\right\}\right|^{2}}{\sum_{l^{\prime}=1}^{L} \sum_{k^{\prime}=1}^{K} \rho_{\mathrm{d} 1} \eta_{l^{\prime} k^{\prime}} \mathbb{E}\left\{\left|\left(\mathbf{g}_{l k}^{l^{\prime}}\right)^{\mathrm{H}} \mathbf{v}_{l^{\prime} k^{\prime}}\right|^{2}\right\}-\rho_{\mathrm{d} 1} \eta_{l k}\left|\mathbb{E}\left\{\left(\mathrm{g}_{l k}^{l}\right)^{\mathrm{H}} \mathbf{v}_{l k}\right\}\right|^{2}+1} .
$$

One can use this lower bound for any choice of precoding vectors and channel estimation schemes. The provided lower bound is tight when the channel hardening level is high; however, for low hardening channel conditions, its performance fluctuates [10, Ch. 4] which should be considered when utilizing this bound.

In this part, we briefly introduced uplink and downlink data transmission for multi-cell Massive MIMO setup. In the papers included in this thesis, we derived some closed-form expressions based on UatF and hardening bound that are provided in this chapter for different Massive MIMO setups and precoding/combining vectors. 


\section{Chapter 3}

\section{Optimization Approaches}

The main objective of this chapter is to briefly introduce some of the primary optimization approaches. Optimization theory is a mathematical tool which is widely used in the wireless communication literature. It is utilized to deal with different resource allocation problems and especially power control problems. In the included papers of this thesis, we formulate different optimization problems with respect to predefined constraints and solve these problems with different optimization methods.

An optimization problem on the standard form is written as $[45, \mathrm{Ch} .4]$

$$
\begin{array}{ll}
\underset{\mathbf{x}}{\operatorname{minimize}} & f_{0}(\mathbf{x}) \\
\text { subject to } & f_{i}(\mathbf{x}) \leq 0, \quad i=1, \ldots, m, \\
& g_{j}(\mathbf{x})=0, \quad j=1, \ldots, q .
\end{array}
$$

In this problem formulation, the vector $\mathbf{x} \in \mathbb{R}^{n}$ denotes the optimization variable (note that $\mathbb{R}^{n}$ is the set of real $n$-length vectors). The cost (or objective) function of this problem is denoted by $f_{0}: \mathbb{R}^{n} \rightarrow \mathbb{R}$ and the $m$ inequality constraints are $f_{i}(\mathbf{x}) \leq 0, \quad i=1, \ldots, m$, in which $f_{i}: \mathbb{R}^{n} \rightarrow \mathbb{R}$ are the inequality constraint functions. In addition, $g_{j}(\mathbf{x})=0, \quad j=1, \ldots, q$, denotes the $q$ equality constraints with the constraint functions $g_{j}: \mathbb{R}^{n} \rightarrow \mathbb{R}[45$, Ch. 4]. The domain of this optimization problem is defined as

$$
\mathcal{D}=\bigcap_{i=0}^{m} \operatorname{dom} \quad f_{i} \quad \cap \bigcap_{j=1}^{q} \operatorname{dom} \quad g_{j},
$$

which is the set of point in which the objective, equality and inequality functions are defined and we write it as the intersection of the domains of the objective and 
all constraints. Note that the $\operatorname{dom} f$ notation is used to denote the domain of each function $f$, i.e., the subset of $\mathbb{R}^{n}$ containing points $\mathbf{x}$ for which $f(\mathbf{x})$ is defined.

The feasible set of this optimization problem is defined as the set of all vectors that belong to $\mathcal{D}$ and satisfies the inequality and equality constraints. A vector $\mathbf{x}^{\text {opt }}$ is a globally optimal solution of this problem if it provides the minimum objective function value among all the points in the feasible set. However, if a vector $\mathbf{x}^{*}$ provides the minimum objective function in the vicinity of itself, this vector is known as the locally minimum solution.

In general, solving an optimization problem to find the globally optimal solution (in case it exists) is a challenging task which depends on many different factors such as the type of cost or constraint functions. However, one can efficiently find the globally optimal solution for some specific sorts of optimization problems that will be described in the following sections.

\subsection{Convex Optimization}

Convex optimization problems are one of the main types of problem formulations that we use in this thesis. One excellent property of a convex problem is that any locally optimal point is also globally optimal. Hence, it is sufficient to design algorithms capable of finding a locally optimal solution. The standard form of a convex optimization problem follows the same formulation as (22) with the additional requirements that the objective function $f_{0}$ and inequality constraint functions $f_{i}, i=1, \ldots, m$ are convex. In addition, to satisfy the convexity requirements, the equality constraint functions $g_{j}, j=1, \ldots, q$ have to be affine. A convex function $f: \mathbb{R}^{n} \rightarrow \mathbb{R}$ is defined such that the domain of $f$ is a convex set (defined as the set such that the line segment of any two points of the set are also included in the set) and for all points $\mathbf{x}_{1}, \mathbf{x}_{2} \in \operatorname{dom} f$, and $0 \leq \alpha \leq 1$ the following holds [45]

$$
f\left(\alpha \mathbf{x}_{1}+(1-\alpha) \mathbf{x}_{2}\right) \leq \alpha f\left(\mathbf{x}_{1}\right)+(1-\alpha) f\left(\mathbf{x}_{2}\right) .
$$

For example $f(x)=x^{2}$ is a convex function. Note that if this equation always holds with equality, the function $f$ is called an affine function and can be drawn as a straight line. The globally optimal solution to a convex optimization problem can be obtained by using, for example, interior-point methods which requires computing the first and second derivative of the objective and constraint functions to update the optimization variable along iterations [45]. In the next part, we introduce linear programming as a special case of convex optimization problems. 


\subsection{Linear Programming}

Linear programming is a fundamental optimization class defined as [45]

$$
\begin{array}{ll}
\underset{\mathbf{x}}{\operatorname{minimize}} & \mathbf{c}^{\mathrm{T}} \mathbf{x} \\
\text { subject to } & \mathbf{a}_{i}^{\mathrm{T}} \mathbf{x} \leq b_{i}, \quad i=1, \ldots, m,
\end{array}
$$

where $\mathbf{c} \in \mathbb{R}^{n}$ and $\mathbf{a}_{i} \in \mathbb{R}^{n}$ are the corresponding coefficients for the objective and constraint functions, respectively. One can map this optimization problem to the standard form in (22) as $f_{0}(\mathbf{x})=\mathbf{c}^{\mathrm{T}} \mathbf{x}$ for the objective function and $f_{i}(\mathbf{x})=$ $\mathbf{a}_{i}^{\mathrm{T}} \mathbf{x}-b_{i}, \forall i$ for the inequality constraint functions. Note that $f_{0}$ and $f_{i}$ 's are linear functions of $\mathbf{x}$. In linear programming, we are minimizing a linear objective function with respect to linear constraints which is the reason for calling this type of problems linear programming.

The linear programming problem generally does not have a simple analytical formula which provides a solution for it [45]. However, it is a convex problem, and there exist several effective methods that solve it in polynomial time. The computation complexity of solving linear programming is relatively low (i.e., in order of $n^{2} m$ assuming $m \geq n$ ) because we do not need to compute the second derivative. In general, we can use general-purpose optimization solving toolbox such as CVX which applies interior-point methods for solving linear programming [46].

\subsection{Epigraph Form}

One can introduce an equivalent epigraph form for the original problem (22):

$$
\begin{array}{cl}
\underset{\mathbf{x}, \lambda}{\operatorname{minimize}} & \lambda \\
\text { subject to } & f_{0}(\mathbf{x})-\lambda \leq 0, \\
& f_{i}(\mathbf{x}) \leq 0, \quad i=1, \ldots, m, \\
& g_{j}(\mathbf{x})=0, \quad j=1, \ldots, q,
\end{array}
$$

with $\mathbf{x} \in \mathbb{R}^{n}$ and $\lambda \in \mathbb{R}$. This new problem is equivalent to the original problem, and besides, it has a more tractable structure as it has a linear objective function, which makes it easier to be solved efficiently. We can solve the equivalent epigraph problem instead of the original problem, and the optimal solution is the solution for the original problem [45].

We applied this type of problem reformulation for solving max-min fairness power control problems in the included papers in this thesis. Note that a detailed explanation of max-min fairness is provided in the next chapter. 


\subsection{Geometric Programming}

Another type of optimization problem that we used for developing our power control algorithm in this thesis is called geometric programming. A standard geometric program has the following form [47-49]

$$
\begin{array}{ll}
\underset{\mathbf{x}}{\operatorname{minimize}} & \sum_{k=1}^{K_{0}} a_{0 k} \prod_{l=1}^{n} x_{l}^{c_{0 k}^{l}} \\
\text { subject to } & \sum_{k=1}^{K_{i}} a_{i k} \prod_{l=1}^{n} x_{l}^{c_{i k}^{l}} \leq 1, \quad i=1, \ldots, m, \\
& a_{j} \prod_{l=1}^{n} x_{l}^{c_{j}^{l}}=1, \quad j=1, \ldots, q,
\end{array}
$$

where $a_{0 k}, a_{i k}, a_{j}$ are positive coefficients and the exponents $c_{0 k}^{l}, c_{i k}^{l}, c_{j}^{l} \in \mathbb{R}$. In this problem formulation, $x_{l} \in \mathbb{R}_{+}$is one of the elements of the vector $\mathbf{x}=$ $\left[x_{1}, \ldots, x_{n}\right]^{\mathrm{T}} \in \mathbb{R}_{+}^{n}$. To map this problem to the standard form provided in (22), we have

$$
\begin{gathered}
f_{0}(\mathbf{x})=\sum_{k=1}^{K_{0}} a_{0 k} \prod_{l=1}^{n} x_{l}^{c_{0 k}^{l}} \\
f_{i}(\mathbf{x})=\sum_{k=1}^{K_{i}} a_{i k} \prod_{l=1}^{n} x_{l}^{c_{i k}^{l}}-1, \forall i \\
g_{j}(\mathbf{x})=a_{j} \prod_{l=1}^{n} x_{l}^{c_{j}^{l}}-1, \forall j .
\end{gathered}
$$

The type of function used in the objective function and inequality constraints is known as a posynomial. The type of function provided in the equality constraint is called a monomial function. Geometric programming in the standard form provided in (26) is a non-convex problem, but can be rewritten as a convex problem. One can apply changes of variables as $x_{l}=e^{y_{l}}$ for all the variables $x_{l}$. In addition, by replacing the objective and inequality constraint functions with their corresponding logarithms, they become logarithms of the sums of the exponential function, which are convex. Besides, taking the logarithm of monomial constraints convert them to linear constraints. The reformulated problem by applying the mentioned changes 
is given by

$$
\begin{aligned}
& \underset{y}{\operatorname{minimize}} \log \left(\sum_{k=1}^{K_{0}} a_{0 k} e^{\sum^{n=1} y_{l} c_{0 k}^{l}}\right) \\
& \text { subject to } \log \left(\sum_{k=1}^{K_{i}} a_{i k} e^{\sum_{l=1}^{n} y_{l} c_{i k}^{l}}\right) \leq 0, \quad i=1, \ldots, m \text {, } \\
& \log \left(a_{j} e^{\sum_{l=1}^{n} y_{l} c_{j}^{l}}\right)=0, \quad j=1, \ldots, q .
\end{aligned}
$$

Since the new reformulated problem is convex, the globally optimal solution can be found efficiently by using a general-purpose optimization solving toolbox such as CVX $[46,47]$. This type of optimization problem is useful for developing proportional fairness power control schemes and such schemes are proposed in the included papers in this thesis. In Paper A, we develop a power control framework for a D2D underlaid Massive MIMO system. In the first part of the paper, geometric programming is utilized to model the proportional fairness power control framework that deals with optimizing data transmission power of D2D and cellular communications at the same time. In addition, in Paper B, we investigate the scalability issue of available power control frameworks for conventional multicell Massive MIMO system. In this paper, geometric programming is used to model proportional fairness power control as well.

\subsection{Signomial Programming}

Signomial programming is an optimization problem that has a similar form as the geometric program provided in (26) but it has the following form

$$
\begin{array}{ll}
\underset{\mathbf{x}}{\operatorname{minimize}} & \sum_{k=1}^{K_{0}} a_{0 k} \prod_{l=1}^{n} x_{l}^{c_{0 k}^{l}} \\
\text { subject to } & \sum_{k=1}^{K_{i}} a_{i k} \prod_{l=1}^{n} x_{l}^{c_{l}^{l}} \leq 1, \quad i=1, \ldots, m, \\
& a_{j} \prod_{l=1}^{n} x_{l}^{c_{j}^{l}}=1, \quad j=1, \ldots, q,
\end{array}
$$

where unlike the geometric programming $a_{0 k}, a_{i k}, a_{j}$ can have negative values as well. Consequently, in this type of optimization problems, the objective and constraint functions can be signomial functions. This problem is non-convex if at least one of the coefficients is negative, and the non-convex problem does not have 
any hidden convex structure as a geometric program has. Solving this non-convex optimization problem has high computational complexity [49]. Therefore, it is more practical to find a locally optimal solution instead. We develop a successive approximation approach in Paper A that provides a local optimal solution for signomial problems efficiently [50]. This type of optimization problems are utilized to model proportional fairness power control when optimizing both pilot and data power coefficients. 


\section{Chapter 4}

\section{Power Control Schemes For Massive MIMO}

In a single-input-single-output (SISO) communication or in a MIMO system with perfect CSI available, one can claim that the transmitter can transmit data with full power to maximize its performance. This is because there is no interference in the SISO case, and for the case of MIMO with perfect CSI, applying beamforming techniques such as zero-forcing (ZF) can offer orthogonal transmission without interference. However, in practical implementations, only imperfect CSI is available, leading to mutual interference in the system. Interference is a limiting factor in wireless communications and occurs due to the broadcast nature associated with wireless propagation channels.

Power control is a fundamental design part of wireless cellular networks. It accommodates cellular networks to control the interference level of wireless links effectively. Consequently, power control directly influences the SE that each user achieves both in the uplink and downlink data transmissions [51]. It is likewise a crucial management part to improve the EE of cellular networks as it helps to minimize the energy consumption of both cellular devices and BSs while guaranteeing the required quality of service of each communication link. Power control has a long history in wireless cellular networks [52,53]. This chapter discusses and explains some of the known power control schemes for Massive MIMO systems.

To explain the basic behaviors of power control, we first investigate two different power control schemes. Figure 5 plots the cumulative distribution function (CDF) of the SE achieved by randomly located users in a multi-cell Massive MIMO setup for two power control schemes during uplink data transmission. In the first case, all users in the network transmit with full power, and in the second case, 
we apply a power control scheme that we call A. We neglect the details of the simulation setup and schemes here to focus on the behaviors. Comparing the two plots, one can see that around 75 percent of the users get a higher SE when we apply scheme A in comparison with full power transmission. However, approximately 25 percent of users in the upper parts of the curves get a higher SE when using full power transmission. This highlights the fact that power control aims at increasing the overall performance of the whole network by balancing the transmit power and controlling the mutual interference. Note that the users with the lowest SEs benefit the most from power control, while the users with the highest SEs are getting a reduced performance. Hence, there is a nontrivial tradeoff between the performance of different users, and different power control schemes are managing the tradeoff differently.

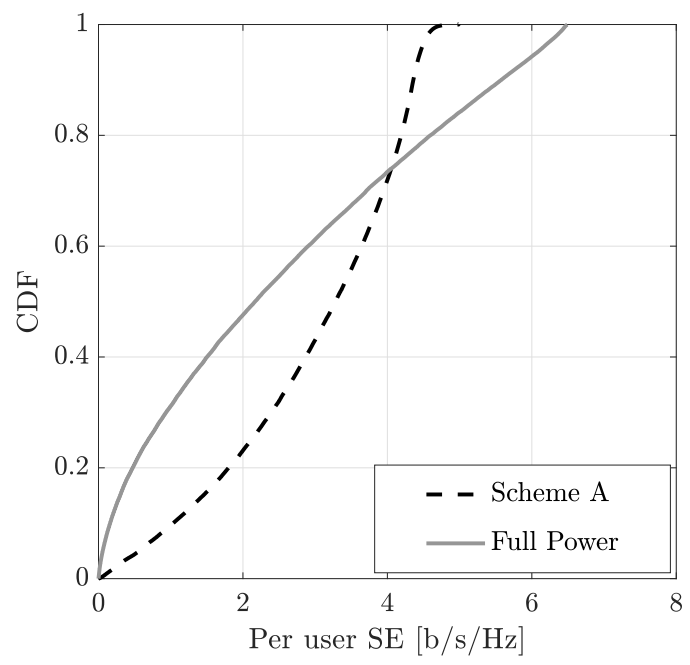

Figure 5: Per user SE for uplink data transmission.

This thesis investigates both uplink and downlink power control schemes with objective functions such as max-min fairness and proportional fairness. These schemes seek to control the mutual interference in the Massive MIMO networks to achieve the optimization objectives. In this chapter, we use the typical multi-cell Massive MIMO system model that is introduced in Chapter 2. First, we discuss the general form of the max-min and proportional fairness power control schemes that have previously appeared in the literature. Then, we provide the main use cases of these power control approaches that are further discussed in the included papers. In Paper A, we extend these power control schemes for D2D underlaid Massive MIMO systems to optimize both data and pilot power of cellular and D2D 
users. We also show that these power control schemes can successfully control additional interference of D2D communication to the conventional Massive MIMO networks. Therefore, applying power control to D2D underlaid Massive MIMO networks eventually increases the sum SE of the whole network. In the second use case, we introduce the scalability issue of max-min and proportional fairness for large multi-cell Massive MIMO systems and describe a new power control scheme to solve this issue which will be further discussed in detail in Paper B.

\subsection{Research Problems on Power Control}

Academic researchers and companies have analyzed and studied Massive MIMO from many different perspectives [40,54-57]. Here, we focus on describing research into power control for Massive MIMO systems. In general, power control is an important design parameter in the cellular networks to mitigate mutual interference among users and enhance the desired part of the received signal at the receivers [51]. When many users are sharing the same time-frequency resources, it is necessary to make tradeoffs between their performance, which can be measured using different metrics. Power control is still an open problem for cellular networks, and as a matter of fact, it has a long research history from single-antenna communication to Massive MIMO systems. Wireless channel fading and the conflicting nature due to co-user interference make power control a challenging design task. Power control algorithms provided in conventional MIMO papers are based on instantaneous channel state information. Therefore they depend on the smallscale fading coefficients. Hence, one should solve and update the transmit powers frequently to track the changes in the small-scale fading. Optimizing the power control based on small-scale fading brings high overhead since frequent control signaling is needed.

The authors in [58] proved that finding a globally optimal power control solution that maximizes sum SE in a multi-user communication system with communication pairs re-using a common frequency band is an NP-hard problem for both uplink and downlink directions which means we can not solve it in polynomial time. Therefore, several research papers have developed power control schemes that achieve a locally optimal solution with a computational complexity that grows polynomially with the number of users [50].

One significant advantage of the channel hardening in Massive MIMO is that the SE expressions depend only on the large-scale channel coefficients representing the average channel quality. Hence, when applying power control to Massive MIMO systems, we only need a long-term power control algorithm as the large-scale fading coefficients change less frequently than small-scale fading. This reduces 
the computational complexity of power allocation at the BSs and also reduces the signaling overhead of the system. As a result, there is a significant computation complexity reduction for solving power control problems in cellular networks equipped with Massive MIMO BSs.

Several researchers have investigated the coexistence of Massive MIMO technology with other existing cellular paradigms [59-61]. D2D communication underlaying cellular networks is one of these paradigms. Massive MIMO can overcome one major problem of D2D underlay communication. The concern is how to control the interference that the D2D underlay communication causes to the cellular network due to sharing the same channel between D2D pairs and cellular users. The massive number of antennas at each BS in Massive MIMO systems handles this thanks to the favorable propagation property.

This motivates us to investigate D2D underlaid multi-cell Massive MIMO systems. The idea is to design a framework supporting D2D pairs coexisting with cellular users in a conventional Massive MIMO setup. Channel estimation plays an essential role in the beamforming design of the Massive MIMO system. It is essential in the D2D underlaid Massive MIMO setup as well. We propose a new pilot transmission scheme and perform channel estimation at both the D2D receiver and the BSs for a D2D underlaid multi-cell Massive MIMO system. Using these channel estimates, we try to find closed-form approximate capacity lower bounds for both D2D and cellular communication, which only depend on the large-scale fading coefficients. Finally, using these SE expressions, we develop power control schemes in D2D underlaid Massive MIMO systems.

In this thesis, we also develop a new power control framework for multi-cell Massive MIMO systems. The framework's target is to overcome a hidden scalability issue of two well-known power control schemes in the Massive MIMO literature, i.e., network-wide max-min fairness and network-wide proportional fairness, which will be discussed in detail in later sections. We explained and introduced a multicell Massive MIMO setup in Chapter 2 and provided a closed-form expression of uplink data transmission for the case of MR combining at the BSs. The following section uses this closed-form expression in our introduction for network-wide max-min and proportional fairness power control schemes in multi-cell Massive MIMO systems. Note that even though we only investigate the case of MR for the uplink data transmission in this chapter, the power control approaches can also be taken for downlink data transmission with MR and other processing schemes. 


\subsection{Max-min Fairness}

This section introduces the general form of the max-min fairness power control scheme for the multi-cell Massive MIMO setup defined in Section 2.3. Max-min fairness is one of the well-known power control schemes suitable for a network serving users with identical data demand. Applying network-wide max-min fairness in the multi-cell Massive MIMO setup provides a uniform SE for all $K \times L$ users. We optimize data power coefficients here to satisfy network-wide max-min fairness criteria. We formulate the optimization problem as

$$
\begin{array}{lll}
\underset{\left\{\eta_{l k}\right\}}{\operatorname{maximize}} & \min _{l, k} & \mathrm{SINR}_{l k} \\
\text { subject to } & 0 \leq \eta_{l k} \leq 1, \forall l, k .
\end{array}
$$

Note that we are maximizing the minimum SINR of the weakest user in the whole network. In the objective function, we replace $\operatorname{SINR}_{l k}$ by the corresponding effective SINR expression provided in (15), which is a function of all the powercontrol coefficients. To solve this problem, we can rewrite it in the epigraph form

$$
\begin{array}{cl}
\underset{\left\{\eta_{l k}\right\}, t}{\operatorname{maximize}} & t \\
\text { subject to } & 0 \leq \eta_{l k} \leq 1, \forall l, k, \\
& \operatorname{SINR}_{l k} \geq t, \forall l, k .
\end{array}
$$

Now, we are maximizing an auxiliary variable $t$ that indicates the SINR requirement of all the users in the network. We have new constraints over SINRs, which correspond to the original objective function. These new constraints are linear constraints for a fixed value of $t$. Hence, to solve this problem, we can fix $t$ and solve the corresponding linear optimization problem, which can be solved efficiently using standard solvers, as described in Subsection 3.2. We can then use the bisection algorithm to perform a line search over the interval $t \in\left[0, t^{\mathrm{u}}\right]$ to find the optimal SINR solution. $t^{\mathrm{u}}$ is an upper bound on the SINRs for which the problem is known to be infeasible. Note that the detailed bisection algorithm for solving the max-min fairness problem is provided in $[10,45]$.

This section briefly described the network-wide max-min fairness power control for a multi-cell Massive MIMO system. We offer a more detailed discussion of this scheme in the included papers. In Paper A, as a practical use case of one of the proposed power control schemes, we extend the network-wide max-min fairness scheme to the case when D2D communication is underlaying a Massive MIMO system. We optimize both data and pilot power coefficients in the proposed scheme, which is more general than what we described here. In Paper B, we investigate 
a hidden scalability issue of network-wide max-min fairness power control in multi-cell Massive MIMO systems and propose a novel scalable solution. Hence, a more detailed study of the max-min fairness power control scheme is available in two of the included papers in this thesis, namely, Paper A and Paper B.

\subsection{Proportional Fairness}

This section concentrates on explaining and discussing the network-wide proportional fairness power control approach for uplink data transmission of a multi-cell Massive MIMO system. Network-wide max-min fairness offers a uniform service for all the users in the network, but it has to sacrifice a lot in SE for an average user to bring up the SE for the weakest user. Therefore, network-wide max-min fairness limits the overall network service performance according to the weakest user. In addition, it cannot satisfy the users with higher service requirements and good channel conditions.

Proportional fairness is a power control scheme that can potentially tackle the limitation mentioned above for network-wide max-min fairness [62]. We write the optimization problem as maximizing the product of the individual SINRs of all users in the network with respect to the power-control coefficient [10]. This objective function is a lower bound on the sum SE of the network. It offers fairness up to some level without sacrificing a lot in SE for an average user. The optimization problem for uplink data transmission of the considered system model is given by

$$
\begin{array}{ll}
\underset{\left\{\eta_{l k}\right\}}{\operatorname{maximize}} & \prod_{l=1}^{L} \prod_{k=1}^{K} \operatorname{SINR}_{l k} \\
\text { subject to } & 0 \leq \eta_{l k} \leq 1, \forall l, k .
\end{array}
$$

This problem can be rewritten by using auxiliary variables $\lambda_{l k}$ in the objective and adding corresponding SINR constraints as [10]

$$
\begin{array}{ll}
\underset{\left\{\eta_{l k}\right\},\left\{\lambda_{l k} \geq 0\right\}}{\operatorname{maximize}} & \prod_{l=1}^{L} \prod_{k=1}^{K} \lambda_{l k} \\
\text { subject to } & 0 \leq \eta_{l k} \leq 1, \forall l, k, \\
& \operatorname{SINR}_{l k} \geq \lambda_{l k}, \forall l, k .
\end{array}
$$

This is an optimization problem with monomial objective function and posynomial constraint. Therefore, it is a geometric programming optimization problem in which it can be solved efficiently by CVX [46].

Here, we provide a numerical comparison of network-wide max-min and proportional fairness algorithms (denoted by NW-MMF and NW-PF in the figures, 
respectively) to highlight their respective behavior. As a baseline, we provide the result for full power transmission denoted as full power in the figures. We consider uplink data transmission in a multi-cell Massive MIMO setup with 9 BSs deployed on a square grid layout with wrap-around to avoid edge effects. Each BS is equipped with $M=100$ antennas and applies MR combining. Furthermore, each BS serves 5 users that are randomly distributed with uniform distribution in the coverage area of the BS. We assume $\tau_{p}=K$ and each coherence block contains 200 symbols. The large-scale fading coefficients are given by [10]

$$
\beta_{l^{\prime}, k}^{l}[\mathrm{~dB}]=-35-36.7 \log _{10}\left(d_{l^{\prime}, k}^{l} / 1 \mathrm{~m}\right)+F_{l^{\prime}, k}^{l}
$$

where $d_{l^{\prime}, k}^{l}$ is the distance between user $k$ located in cell $l^{\prime}$ to BS $l$. In addition, $F_{l^{\prime}, k}^{l}$ is the shadow fading generated from a log-normal distribution with standard deviation $7 \mathrm{~dB}$. The noise variance is set to $-94 \mathrm{dBm}$, and the maximum transmit power of the users is $200 \mathrm{~mW}$ for uplink data transmission [63]. Figure 6 plots the CDF of the SE of all the users for uplink data transmission, for different random user locations with uniform distribution. It can be seen from the figures that

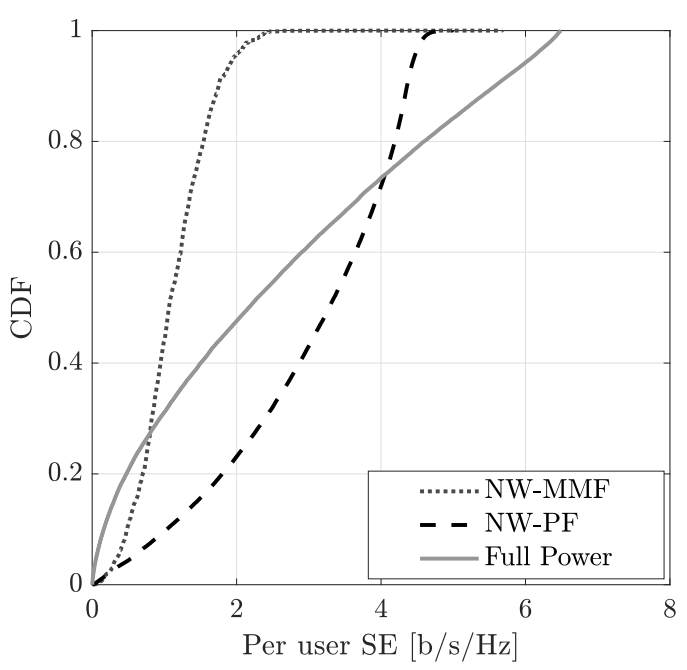

Figure 6: Per user SE for uplink data transmission for different random user locations.

network-wide proportional fairness gives higher SE than max-min fairness for the majority of users. By comparing network-wide max-min and proportional fairness with no power control, we can see that $30 \%$ and $75 \%$ of users get higher SE, respectively. However, by making a one-to-one comparison for all the users, we notice that $12 \%$ of the users get better SE with network-wide max-min fairness 
than with network-wide proportional fairness. It is the users with the lowest SEs that benefit from the max-min fairness objective function.

In Figure 7, we show the CDF of the sum SE in the network with respect to different random user locations. We can see that network-wide proportional fairness performs the best in terms of sum SE, as we mentioned previously. The conclu-

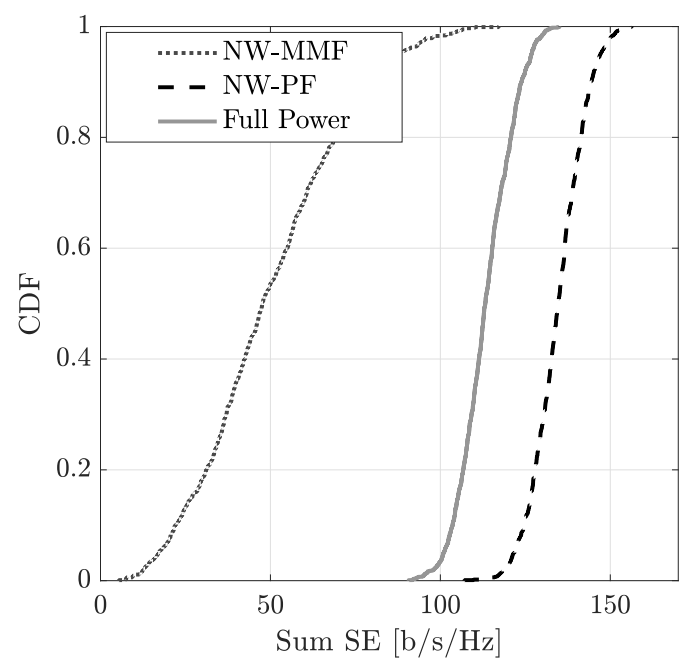

Figure 7: Sum SE for uplink data transmission for different random user locations.

sion is that different power control methods are maximizing different objective functions, and it is up to the network designer to select an appropriate objective function.

\subsection{Use Cases}

This section describes some practical use cases of the power control schemes that are also considered in the first two papers included in this thesis.

\subsubsection{D2D Underlay Communications}

In this subsection, we provide some basic definitions of D2D communications where we mainly focus on D2D underlay Massive MIMO communication and briefly explain data and pilot power control for D2D underlaid Massive MIMO setups. D2D communications is an alternative communication paradigm in wireless cellular systems in which the transmitting and receiving users are offered direct communication instead of sending traffic through the BSs. For D2D communication 
to be effective, the transmitter and receiver must be located in the vicinity of each other. They bypass the BS that results in offloading a portion of the BS's traffic load, which potentially improves the sum SE of the network. There are other gains that D2D offers due to the short range of communication between D2D users. These are for example higher SE for the D2D users and lower power transmission that results in a longer battery life [26,64-67].

Different D2D communication policies are available in the literature, e.g., [23]. There are two main classes of D2D communication. The first one is called inband communication, in which D2D users reuse the cellular resources, and the second one is outband that uses unlicensed spectrum. Outband D2D does not use cellular resources and makes no overhead for the cellular network. However, the service providers do not control the connection and quality of service as it uses the license-free band. Therefore, it has received less attention in the literature. In inband communication, the D2D users can either use the same resources as the cellular users, called D2D underlay communication or get dedicated resources for D2D communication that cellular users do not use. Dedicating cellular resources for D2D communication can decrease the SE of the network; hence, underlay D2D communication is preferable. It can notably enhance the SE and EE of the cellular system since it increases the usage of cellular resources by sharing them for D2D communication. However, sharing cellular resources for D2D underlay communication generates additional interference that needs to be controlled. There are several ways to mitigate the extra interference from D2D to cellular communication and vice versa. For example, mode selection, scheduling techniques, power allocation, and beamforming schemes $[65,67,68]$.

In Paper A, we focus on D2D communication underlaying Massive MIMO systems. We investigate how Massive MIMO BSs helps us to mitigate the extra interference to the network from D2D underlay communication. We also use power control as one of the main tools for interference management for D2D underlay communication. This paper utilizes both the network-wide max-min and proportional fairness power control schemes discussed in Section 4.2 and 4.3, respectively. To be more specific, we derive closed-form SE expressions for the cellular and D2D communications. Then, we use these SE expressions to formulate and solve the proposed power control schemes. However, instead of optimizing only the data power coefficients in this chapter, we propose optimizing both the pilot and data power coefficients. Besides, we propose a new pilot allocation strategy to limit the interference of D2D communication to the cellular system during the channel estimation phase. We show that our new framework enhances the SE of conventional Massive MIMO systems without drastically affecting the performance of cellular users. Consequently, the cellular network serves more users and offers higher aggregate SE for the network. 


\subsubsection{Scalability Issue for Power Control in Multi-cell Massive MIMO}

This subsection highlights the scalability issues of network-wide max-min and proportional fairness power control schemes for the multi-cell Massive MIMO setup.

Both network-wide max-min and proportional fairness power control schemes are beneficial for multi-cell Massive MIMO systems. However, applying networkwide max-min and proportional fairness as the utility function for multi-cell Massive MIMO systems has a significant limitation. Both of these approaches profoundly suffer from scalability issues in large networks. To be more specific, increasing the network size increases the risk of having a user in the network with a very bad channel condition. This situation will penalize the whole system and result in zero overall SE for the system as the large-scale fading coefficient of the weakest user goes to zero. Paper B provides detailed mathematical proofs for the scalability issue of these two power control schemes. To overcome the scalability issue, we propose a novel approach, which builds on the use of a new objective function: maximization of the geometric mean of per cell max-min fairness. We prove that this problem is scalable and can be solved efficiently. 


\section{Chapter 5}

\section{Neural Networks}

The idea of using (artificial) neural networks in computer science evolved based on inspiration from the (biological) neural networks of the human brain, which can be seen as a computational unit consisting of a network of interconnected neurons that can learn from experience by adjusting its underlying connections [69]. In computer science, the process of learning begins with feeding input data to a computer algorithm to analyze the data and find an answer to a question, essentially searching for a mapping between the input data and a known set of potential output data (e.g., representing possible decisions). Machine learning and deep learning are used to discover patterns from raw data.

Conventional machine learning algorithms are highly dependent on expert knowledge to define the features in a specific formalization appropriate for the machine learning algorithm applied. Otherwise, these algorithms cannot find useful patterns in the data. In contrast, deep learning algorithms constitute a more flexible subset of machine learning algorithms and can find different features from the data without human intervention [69]. Many machine learning and deep learning algorithms are many decades old, but most of them were not utilized in products because of a lack of computing resources or enough data. However, due to the recent advancements of super-powerful computing resources and access to a tremendous amount of data, they became very popular in many different fields [70], especially deep learning algorithms gained more attention as they require higher computational power than conventional algorithms. In recent years, there has been an increasing interest in utilizing deep neural networks (DNNs) in different research areas as we observe better performance from DNN, for example, in natural language processing [71], computer vision [72], medical diagnosis [73], etc.

Deep learning algorithms can provide solutions for a class of problems that lack accurate mathematical models to solve them. Some of these problems are easy for 
humans to solve, for instance, finding an object in an image or video. Nevertheless, finding accurate models and algorithms for solving them via computer programs is very complex. However, these complex problems can be solved by off-the-shelf deep learning methods.

We are equipped with many efficient and accurate model-based solutions in wireless commutations. It refers to problems that we have mathematical methods to explicitly solve them. For example, detection, estimation, resource allocation and power control. Nevertheless, in recent years, we face some more complex scenarios that are difficult to handle with model-based methods [74-76]. One of these problems occurs when we have a model deficiency, which means that it is difficult to find physical models, for instance, because they involve human behavior. Some examples of such scenarios are modeling data traffic or the movement of human-used devices in the network. We know that there is a structure in these scenarios, but we cannot model it reasonably. For example, in cellular networks, we know that the network might not be used too much at night or might be used in high demand in some peak hours. However, we do not have a mathematical model for it. Another example is that we do not have a physical model to predict the mobility of the users in the network. Nevertheless, we can learn from the data for both examples and utilize that knowledge to improve our cellular network design, performance, and energy efficiency. As an example, by switching off the idle access points in no traffic hours, we can save a significant amount of grid energy or control some of the access points to only operate during peak hours. The solutions provided based on learning from the data fall in the category of data-driven approches.

Another type of problem occures when we have an algorithmic deficiency. It refers to situations where we already have mathematical models and solutions for solving some specific problems in the wireless networks; however, they are computationally complex. For example, we know that the interior-point method, which is used to solve optimization problems mentioned in Chapter 3, will converge to the global optimum, but this does not mean that it is the most computationally efficient way of finding the optimum. A DNN can be used to solve these types of problems approximately. One approach is called deep unfolding i.e., the DNN is designed to mimic the existing algorithm but has tunable parameters [77]. It utilizes the data to tweak the algorithms to work for the class of problems we are interested in. However, there might exist some other classes of problems where the solution would not work as good. Another potential use case for DNN to solve algorithmic deficiency in wireless communication is facilitating the real-time implementation of high computational complexity iterative problems [78,79]. In these iterative optimization-based algorithms, even though we have access to the algorithms that are providing optimal solutions, real-time implementation is an issue due to 
high computational complexity [75]. It is possible to use the output knowledge from optimization-based algorithms to generate training data for neural networks and train neural networks that are capable of providing reasonable approximate solutions for this problem in real-time. Deep learning techniques can tackle these complex problems, for instance, in networks with many unknowns or "parameters that are hard to estimate."

With the increase in the number of wireless devices, high data rate requirements, and having human behavior involved in the network, we are facing more model and algorithmic deficiencies which is the price to pay for making the systems more efficient. In such complex networks, it is essential to utilize new tools which can help to learn the underlying structure of data. Deep learning has the potential to be used as a complementary tool to the classical mathematical tools available in wireless networks to solve algorithmic deficiency and model deficiency related to the applications of wireless networks [74,80-82]. This chapter provides a brief introduction to deep learning and an application of DNN in channel estimation in Massive MIMO networks.

\subsection{Basics of Deep Learning}

Having access to more data and powerful computational resources motivates us to use the potential of DNNs to solve computationally complex problems related to the design and applications of wireless networks from the data. Artificial neural networks based methods are inspired by the neural network structure of human brains to model the underlying mapping function $y=f\left(d_{0} ; \theta\right)$ of a system with $m$-length input data vector $\mathbf{d}_{0} \in \mathbb{R}^{n_{0}}$ and its corresponding $n_{L}$-length output vector of $\mathbf{y} \in \mathbb{R}^{n_{L}}$ for a parameter vector $\theta[69,83]$. In this section, we briefly explain a well-known neural network structure known as the fully-connected feed-forward network architecture. In this type of structure, the neural network consists of $L-1$ hidden layers, meaning they are private to the neural network and hidden from external systems. Function $\mathrm{f}$ is a composition of $L$ layered functions $\mathrm{f}_{1}, \ldots, \mathrm{f}_{L}$ that describe the flow of input data through the neurons in the hidden layers towards the output. Each of the $f_{1}, \ldots, f_{L}$ functions associated to one hidden layer and shows the mapping from one layer to the next [69]. Figure 8 depicts a fully-connected feed-forward neural network with $L=3$ layers.

The term DNN refers to neural networks with several hidden layers. Each layer's function is modeled as [83]

$$
\mathbf{d}_{l}=\mathrm{f}_{l}\left(\mathbf{d}_{l-1} ; \boldsymbol{\theta}_{l}\right)=\sigma_{l}\left(\mathbf{W}_{l} \mathbf{d}_{l-1}+\mathbf{b}_{l}\right),
$$

where function $\mathrm{f}_{l}$ is determined by a set of parameters $\boldsymbol{\theta}_{l}$ [83]. In addition, $\mathbf{d}_{l-1}$ denotes the output vector of hidden layer $l-1$ which goes through an affine 


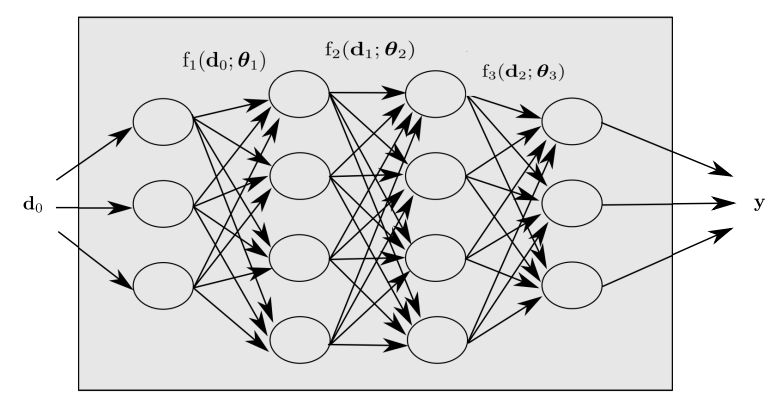

Figure 8: A representation of a fully-connected feed-forward neural network with $L=3$.

transformation by multiplying it with the weight matrix of layer $l$, i.e., $\mathbf{W}_{l} \in$ $\mathbb{R}^{n_{l} \times n_{l-1}}$ and adding the bias vector $\mathbf{b}_{l}$. We define the set of parameters for each layer as $\boldsymbol{\theta}_{l}=\left\{\mathbf{W}_{l}, \mathbf{b}_{l}\right\}$. A nonlinear activation function $\sigma_{l}$ will be applied to the corresponding value from the affine transformation at each neuron of layer $l$. Note that nonlinear activation function facilitate to learn more complex structures from the data. The selection of activation function for each hidden layer is one of the design aspects of DNNs. The activation functions are fixed, while $\boldsymbol{\theta}$ is tuned during the learning process. This allow us to approximate different functions, that all belong to a certain class determined by the architecture.

For the generic neural network with $L-1$ hidden layers, we have

$$
y=f_{L}\left(f_{L-1}\left(\ldots\left(f_{2}\left(f_{1}\left(d_{0} ; \theta_{1}\right) ; \theta_{2}\right) \ldots\right) ; \theta_{L-1}\right) ; \theta_{L}\right),
$$

where $\boldsymbol{\theta}=\left\{\boldsymbol{\theta}_{1}, \ldots, \boldsymbol{\theta}_{L}\right\}$ is defined as the set of all parameters of the network. The propagation of input data via the hidden layers to the output is known as forward propagation.

Suppose we have a specific set of input data (observations), and we know that there exists a function that maps it to a specific output data. However, we do not know the function. We can use neural networks to approximate the underlying mapping function. Based on the universal approximation theorem [84], there exists a fully connected feed-forward neural network with one hidden layer that can approximate any continuous function to any given accuracy if just the number of parameters is sufficiently large. This network consists of a finite number of neurons, weight, and bias parameters.

Here, we focus on training the DNN via a class of machine learning known as supervised learning. In supervised learning, we have a training data set which consists of input data vectors $\mathbf{d}_{t}$, with the corresponding outputs $\mathbf{y}_{t}=g\left(\mathbf{d}_{t}\right)$, called labels. The function $g($.$) is an unknown mapping function. We use the training$ data set to find a set of parameters $\hat{\boldsymbol{\theta}}$ that minimizes some predefined loss function. 
The loss function indicates the accuracy of our approximation for the mapping function. We consider a loss function as

$$
L(\boldsymbol{\theta})=\frac{1}{T} \sum_{t=1}^{T}\left|\mathrm{y}_{t}-\mathrm{f}\left(\mathbf{d}_{t} ; \boldsymbol{\theta}\right)\right|^{2},
$$

where $\left(\mathbf{d}_{t}, \mathbf{y}_{t}\right)$ indicates a pair of input and output in the labeled training data set, the training data set consists of $T$ different pairs of input and output samples. The loss function is used to measure the approximation mismatch. One can apply iterative approaches such as stochastic gradient descent (SGD) to iteratively optimize parameters that approximate the mapping function of training data to get an acceptable solution with low approximation error. This update process for weight and bias parameters of neural networks based on the current loss function is called back propagation [69]. In SGD, we randomly initialize a set of parameters $\boldsymbol{\theta}=\boldsymbol{\theta}_{0}$ which refers to setting initial values for weights and biases of each hidden layer. The idea is to update $\boldsymbol{\theta}$ iteratively on random mini-batches (subsets) of training data according to the following policy of SGD during the training [69]

$$
\boldsymbol{\theta}_{i+1}=\boldsymbol{\theta}_{i}-\alpha \nabla L\left(\boldsymbol{\theta}_{i}\right)
$$

where $0<\alpha<1$ is the learning rate. Note that using mini-batches helps to reduce the computational complexity while calculating gradients. The ultimate goal of training in DNN is that the trained network should be generalizable. It means that one can use $\hat{\theta}$ and function $f$, to approximate the correct output not only on the training data but also on any other input data vector generated from the same distribution as the training input data. One can use a part of the training data set (known as validation set) to evaluate how well the DNN predicts the output and compare it with the true output. This can help to measure the performance of DNN during the training phase itself and fine-tune some of the network's parameters, such as the number of nodes per hidden layer, learning rate, etc., which are called hyper-parameters.

After we have trained our DNN, we can use another set of data known as the test dataset to further evaluate the performance of the trained network by comparing the predicted output with the true output. The idea is to evaluate the performance of the trained network on a new data set coming from the same distribution that we expect to see in the input. We tend to investigate how the network generalizes to previously not observed data. This is called generalization. The objective is to minimize the expected generalization error. There are many factors and hyperparameters that play a role in the design of a DNN structure. Furthermore, the neural network structure depends on the type of data and its nature depending upon the environment, scenario, applications, and complexity. The detailed explanation 
of different types of network structure and hyper-parameter design is out of the scope of this chapter. For more detailed information, it can be found in [69]. To design and implement a neural network, one can use many open-source, powerful publicly available libraries.

\subsection{DNN for Channel Estimation}

In this section, the main idea is to briefly discuss the utilization of DNN as a datadriven approach to perform channel estimation in Massive MIMO. In general, the idea of estimation is to find a good approximate value of an unknown variable from measurement data. For the case that the unknown variable is a realizations of a random variable with known statistical properties, the problem is categorized in the class of Bayesian estimation. This is of our interest as in wireless communication, the channels are the realization of some random variables. In Bayesian estimation, we need to know the statistical distribution, this is a common assumption for Massive MIMO networks. It is shown in [10] that how one can obtain them. In Massive MIMO networks, to fully benefit from having many antennas at the BSs and perform resource allocation, each BS must have access to a high-quality channel estimation from the active users that it is going to serve in the current coherence interval. The channel estimates can be acquired via the uplink pilot transmission phase, which is discussed in Section 2.4.

Upon receiving a signal from downlink data transmission of BS, the user must know their effective channel gain to decode the desired data. One common practice is to use the statistical mean of effective channel gains as the estimate in Massive MIMO networks in rich scattering environments which offer channel hardening. However, in low-hardening conditions, using channel statistics causes fluctuation in the performance. To tackle this issue, we first investigate a model-based approach based on blind channel estimation, which uses the average received signal power during downlink data to estimate effective channel gains. This method is based on the asymptotic properties of the system model, such as having a large coherence interval or having many active users per cell, a more detailed discussion is provided in Paper C. However, there is no guarantee for these asymptotic properties in practical Massive MIMO networks as they might have few active users or a short coherence interval.

Another possible approach is to use DNN to estimate downlink effective channel gains in multi-cell massive Massive MIMO Networks. Deep learning can be used to "learn" an estimator that extracts the statistics from the data. In addition, the loss function determines what kind of estimator it will be. If the MSE is minimized, the unknown mapping function $g($.$) is the true MMSE estimator, and we will learn an$ 
approximation of it. Hence, as an alternative, we investigate how to improve the performance of the Massive MIMO network by empowering the user to estimate the downlink channel from downlink data transmissions utilizing a data-driven approach based on neural networks. This approach is not conditioned on the asymptotic properties of the system model. In Paper C, we discuss both approaches in detail and provide a performance comparison between them and state-of-the-art. 


\section{Bibliography}

[1] D. H. Ring, "Mobile telephony-wide area coverage," Bell Laboratories Technical Memorandum, 1947.

[2] R. H. Frenkiel, "A high-capacity mobile radiotelephone system model using a coordinated small-zone approach," IEEE Transactions on Vehicular Technology, vol. 19, no. 2, pp. 173-177, 1970.

[3] V. H. M. Donald, "Advanced mobile phone service: The cellular concept," The Bell System Technical fournal, vol. 58, no. 1, pp. 15-41, 1979.

[4] W. R. Young, "Advanced mobile phone service: Introduction, background, and objectives," The Bell System Technical fournal, vol. 58, no. 1, pp. 1-14, 1979.

[5] J. G. Andrew, S. Buzzi, W. Choi, S. V. Hanly, A. Lozano, A. C. K. Soong, and J. C. Zhang, "What will 5G be?" IEEE fournal on Selected Areas in Communications, vol. 32, no. 6, pp. 1065-1082, 2014.

[6] M. Series, IMT Vision-Framework and overall objectives of the future development of IMT for 2020 and beyond, Std., 2015.

[7] Cisco, "Visual networking index: Global mobile data traffic forecast update, 2017-2022 white paper," Tech. Rep., 2019.

[8] Ericsson, "Ericsson mobility report," Tech. Rep., 2020. [Online]. Available: https://www.ericsson.com/en/mobility-report/reports/november-2020

[9] S. Parkvall, E. Dahlman, A. Furuskar, and M. Frenne, "NR: The new 5G radio access technology," IEEE Communications Standards Magazine, vol. 1, no. 4, pp. 24-30, 2017.

[10] E. Björnson, J. Hoydis, and L. Sanguinetti, Massive MIMO Networks: Spectral, Energy, and Hardware Efficiency. Now Publishers, Inc., 2017, vol. 11, no. 3-4.

[11] V. Jungnickel, K. Manolakis, W. Zirwas, B. Panzner, V. Braun, M. Lossow, M. Sternad, R. Apelfröjd, and T. Svensson, "The role of small cells, coordinated multipoint, and massive MIMO in 5G," IEEE Communications Magazine, vol. 52, no. 5, pp. 44-51, 2014.

[12] V. Chandrasekhar, J. G. Andrews, and A. Gatherer, "Femtocell networks: a survey," IEEE Communications Magazine, vol. 46, no. 9, pp. 59-67, 2008. 
[13] M. Dohler, R. W. Heath, A. Lozano, C. B. Papadias, and R. A. Valenzuela, "Is the PHY layer dead?" IEEE Communications Magazine, vol. 49, no. 4, pp. 159-165, 2011.

[14] T. L. Marzetta, "Noncooperative cellular wireless with unlimited numbers of base station antennas," IEEE Transactions on Wireless Communications, vol. 9, no. 11, pp. 3590-3600, 2010.

[15] F. Boccardi, R. W. Heath, A. Lozano, T. L. Marzetta, and P. Popovski, "Five disruptive technology directions for 5G," IEEE Communications Magazine, vol. 52, no. 2, pp. 74-80, 2014.

[16] C.-X. Wang, F. Haider, X. Gao, X.-H. You, Y. Yang, D. Yuan, H. M. Aggoune, H. Haas, S. Fletcher, and E. Hepsaydir, "Cellular architecture and key technologies for $5 \mathrm{G}$ wireless communication networks," IEEE Communications Magazine, vol. 52, no. 2, pp. 122-130, 2014.

[17] T. V. Chien and E. Björnson, "Massive MIMO communications," in 5G Mobile Communications. Springer, 2017, pp. 77-116.

[18] F. Rusek, D. Persson, B. K. Lau, E. G. Larsson, T. L. Marzetta, O. Edfors, and F. Tufvesson, "Scaling up MIMO: Opportunities and challenges with very large arrays," IEEE Signal Processing Magazine, vol. 30, no. 1, pp. 40-60, 2012.

[19] E. Björnson, E. G. Larsson, and T. L. Marzetta, "Massive MIMO: Ten myths and one critical question," IEEE Communications Magazine, vol. 54, no. 2, pp. 114-123, 2016.

[20] H. Q. Ngo, "Massive MIMO: Fundamentals and system designs," PhD disseratation, Linköping University, 2015.

[21] X. Lin, R. W. Heath, and J. G. Andrews, "The interplay between massive MIMO and underlaid D2D networking," IEEE Transactions on Wireless Communications, vol. 14, no. 6, pp. 3337-3351, 2015.

[22] A. Ghazanfari, "Coordinated beamforming and power control for network controlled device-to-device (D2D) communication," University of Oulu, 2014.

[23] A. Asadi, Q. Wang, and V. Mancuso, "A survey on device-to-device communication in cellular networks," IEEE Communications Surveys \& Tutorials, vol. 16, no. 4, pp. 1801-1819, 2014.

[24] A. Ghazanfari, A. Tölli, and H. Pennanen, "Sum power minimization for cellular systems with underlay D2D communications," in 9th International 
Conference on Cognitive Radio Oriented Wireless Networks and Communications (CROWNCOM). IEEE, 2014, pp. 45-50.

[25] X. Lin, J. Andrews, A. Ghosh, and R. Ratasuk, "An overview of 3GPP deviceto-device proximity services," IEEE Communications Magazine, vol. 52, no. 4, pp. 40-48, 2014.

[26] A. Ghazanfari, A. Tölli, and J. Kaleva, "Joint power loading and mode selection for network-assisted device-to-device communication," in IEEE International Conference on Communications (ICC), 2015, pp. 2548-2553.

[27] T. L. Marzetta, E. G. Larsson, H. Yang, and H. Q. Ngo, Fundamentals of massive MIMO. Cambridge University Press, 2016.

[28] D. Tse and P. Viswanath, Fundamentals of wireless communication. Cambridge university press, 2005.

[29] R. W. H. Jr and A. Lozano, Foundations of MIMO communication. Cambridge University Press, 2018.

[30] A. Goldsmith, S. A. Jafar, N. Jindal, and S. Vishwanath, "Capacity limits of MIMO channels," IEEE fournal on Selected Areas in Communications, vol. 21, no. 5, pp. 684-702, 2003.

[31] D. Gesbert, M. Kountouris, R. W. H. Jr, C.-B. Chae, and T. Sälzer, "From single user to multiuser communications: shifiting the MIMO paradigm," IEEE Signal Processing Magazine, 2008.

[32] G. Caire and S. Shamai, "On the achievable throughput of a multiantenna gaussian broadcast channel," IEEE Transactions on Information Theory, vol. 49, no. 7, pp. 1691-1706, 2003.

[33] T. L. Marzetta, "Massive MIMO: An introduction," Bell Labs Technical fournal, vol. 20, pp. 11-22, 2015.

[34] H. Q. Ngo, E. G. Larsson, and T. L. Marzetta, "Aspects of favorable propagation in massive MIMO," in 22nd European Signal Processing Conference (EUSIPCO). IEEE, 2014, pp. 76-80.

[35] X. Wu, N. C. Beaulieu, and D. Liu, "On favorable propagation in massive MIMO systems and different antenna configurations," IEEE Access, vol. 5, pp. 5578-5593, 2017. 
[36] B. M. Hochwal, T. L. Marzetta, and V. Tarokh, "Multiple-antenna channel hardening and its implications for rate feedback and scheduling," IEEE Transactions on Information Theory, vol. 50, no. 9, pp. 1893-1909, 2004.

[37] S. Gunnarsson, J. Flordelis, L. V. der, and F. Tufvesson, "Channel hardening in massive MIMO: Model parameters and experimental assessment," IEEE Open fournal of the Communications Society, vol. 1, pp. 501-512, 2020.

[38] E. Björnson, L. Sanguinetti, J. Hoydis, and M. Debbah, "Designing multi-user MIMO for energy efficiency: When is massive MIMO the answer?" in IEEE Wireless Communications and Networking Conference (WCNC). IEEE, 2014, pp. 242-247.

[39] S. K. Mohammed and E. G. Larsson, "Per-antenna constant envelope precoding for large multi-user MIMO systems," IEEE Transactions on Communications, vol. 61, no. 3, pp. 1059-1071, 2013.

[40] J. Hoydis, S. T. Brink, and M. Debbah, "Massive MIMO in the UL/DL of cellular networks: How many antennas do we need?" IEEE Journal on selected areas in communications, vol. 31, no. 2, pp. 160-171, 2013.

[41] C. Shepard, H. Yu, N. Anand, E. Li, T. L. Marzetta, R. Yang, and L. Zhong, "Argos: Practical many-antenna base stations," in 18th Annual International Conference on Mobile Computing and Networking, 2012, pp. 53-64.

[42] J. Vieira, S. Malkowsky, K. Nieman, Z. Miers, N. Kundargi, L. Liu, I. Wong, V. Öwall, O. Edfors, and F. Tufvesson, "A flexible 100-antenna testbed for massive MIMO," in Proceedings of IEEE Global Communications (GLOBECOM) Workshops. IEEE, 2014, pp. 287-293.

[43] H. Q. Ngo and E. G. Larsson, "No downlink pilots are needed in TDD massive MIMO," IEEE Transactions on Wireless Communications, vol. 16, no. 5, pp. 2921-2935, 2017.

[44] S. M. Kay, Fundamentals of statistical signal processing, 1993.

[45] S. Boyd and L. Vandenberghe, Convex Optimization, New York, NY, USA, 2004.

[46] M. Grant, S. Boyd, and Y. Ye, "CVX: Matlab software for disciplined convex programming," 2008.

[47] S. Boyd, S.-J. Kim, L. Vandenberghe, and A. Hassibi, "A tutorial on geometric programming," Optimization and engineering, vol. 8, no. 1, p. 67, 2007. 
[48] R. J. Duffin, "Geometric programming-theory and application,” Tech. Rep., 1967.

[49] K. Lange and H. Zhou, "MM algorithms for geometric and signomial programming," Mathematical Programming, vol. 143, no. 1, pp. 339-356, 2014. [Online]. Available: https://doi.org/10.1007/s10107-012-0612-1

[50] M. Chiang, C. W. Tan, D. P. Palomar, D. O'neill, and D. Julian, "Power control by geometric programming," IEEE Transactions on Wireless Communications, vol. 6, no. 7, pp. 2640-2651, 2007.

[51] M. Chiang, P. Hande, T. Lan, and C. W. Tan, "Power control in wireless cellular networks," Foundations and Trends® in Networking, vol. 2, no. 4, pp. 381-533, 2008 .

[52] F. Bock and B. Ebstein, "Assignment of transmitter powers by linear programming," IEEE Transactions on Electromagnetic Compatibility, vol. 6, no. 2, pp. 36-44, 1964.

[53] J. Zander, "Performance of optimum transmitter power control in cellular radio systems," IEEE Transactions on Vehicular Technology, vol. 41, no. 1, pp. 57-62, 1992.

[54] E. G. Larsson, O. Edfors, F. Tufvesson, and T. L. Marzetta, "Massive MIMO for next generation wireless systems," IEEE Communications Magazine, vol. 52, no. 2, pp. 186-195, 2014.

[55] L. Lu, G. Y. Li, A. L. Swindlehurst, A. Ashikhmin, and R. Zhang, "An overview of massive MIMO: Benefits and challenges," IEEE Journal of Selected Topics in Signal Processing, vol. 8, no. 5, pp. 742-758, 2014.

[56] H. Q. Ngo, E. G. Larsson, and T. L. Marzetta, "Energy and spectral efficiency of very large multiuser MIMO systems," IEEE Transactions on Communications, vol. 61, no. 4, pp. 1436-1449, 2013.

[57] H. Yang and T. L. Marzetta, "Performance of conjugate and zero-forcing beamforming in large-scale antenna systems," IEEE Journal on Selected Areas in Communications, vol. 31, no. 2, pp. 172-179, 2013.

[58] Z.-Q. Luo and S. Zhang, "Dynamic spectrum management: Complexity and duality," IEEE Journal on Selected Areas in Communications, vol. 2, no. 1, pp. 57-73, 2008. 
[59] G. Yang, C. K. Ho, R. Zhang, and Y. L. Guan, "Throughput optimization for massive MIMO systems powered by wireless energy transfer," IEEE fournal on Selected Areas in Communications, vol. 33, no. 8, pp. 1640-1650, 2015.

[60] Z. Gao, L. Dai, D. Mi, Z. Wang, M. A. Imran, and M. Z. Shakir, "MmWave massive-MIMO-based wireless backhaul for the $5 \mathrm{G}$ ultra-dense network," IEEE Wireless Communications, vol. 22, no. 5, pp. 13-21, 2015.

[61] J. Hoydis, K. Hosseini, S. ten Brink, and M. Debbah, "Making smart use of excess antennas: Massive MIMO, small cells, and TDD," Bell Labs Technical fournal, vol. 18, no. 2, pp. 5-21, 2013.

[62] F. Kelly, A. Maulloo, and D. Tan, "Rate control for communication networks: Shadow prices, proportional fairness and stability," f. Operational Research Society, vol. 49, no. 3, pp. 237-252, 1997.

[63] A. Ghazanfari, H. V. Cheng, E. Björnson, and E. G. Larsson, "A fair and scalable power control scheme in multi-cell massive MIMO," in IEEE International Conference on Acoustics, Speech and Signal Processing (ICASSP), 2019, pp. 44994503.

[64] K. Doppler, C.-H. Yu, C. B. Ribeiro, and P. Janis, "Mode selection for deviceto-device communication underlaying an LTE-advanced network," in IEEE Wireless Communication and Networking Conference. IEEE, 2010, pp. 1-6.

[65] C.-H. Yu, K. Doppler, C. B. Ribeiro, and O. Tirkkonen, "Resource sharing optimization for device-to-device communication underlaying cellular networks," IEEE Transactions on Wireless Communications, vol. 10, no. 8, pp. 2752-2763, 2011.

[66] K. Doppler, M. Rinne, C. Wijting, C. B. Ribeiro, and K. Hugl, "Device-todevice communication as an underlay to LTE-advanced networks," IEEE Communications Magazine, vol. 47, no. 12, pp. 42-49, 2009.

[67] H. Min, W. Seo, J. Lee, S. Park, and D. Hong, "Reliability improvement using receive mode selection in the device-to-device uplink period underlaying cellular networks," IEEE Transactions on Wireless Communications, vol. 10, no. 2, pp. 413-418, 2010.

[68] X. Tao, X. Xiao, and J. Lu, "A QoS-aware power optimization scheme in OFDMA systems with integrated device-to-device (D2D) communications," IEEE Vehicular Technology Conference (VTC), 2012. 
[69] I. Goodfellow, Y. Bengio, and A. Courville, Deep learning. MIT press Cambridge, 2016, vol. 1, no. 2.

[70] Y. LeCun, Y. Bengio, and G. Hinton, "Deep learning," Nature, vol. 521, no. 7553, pp. 436-444, 2015.

[71] T. Young, D. Hazarika, S. Poria, and E. Cambria, "Recent trends in deep learning based natural language processing," IEEE Computational Intelligence Magazine, vol. 13, no. 3, pp. 55-75, 2018.

[72] A. Voulodimos, N. Doulamis, A. Doulamis, and E. Protopapadakis, "Deep learning for computer vision: A brief review," Computational Intelligence and Neuroscience, vol. 2018, 2018.

[73] D. Shen, G. Wu, and H.-I. Suk, "Deep learning in medical image analysis," Annual Review of Biomedical Engineering, vol. 19, pp. 221-248, 2017.

[74] L. Zhang and Ying-ChangLiang, "Deep reinforcement learning for multi-agent power control in heterogeneous networks," IEEE Transactions on Wireless Communications, 2020.

[75] W. Xia, G. Zheng, Y. Zhu, J. Zhang, J. Wang, and A. P. Petropulu, "A deep learning framework for optimization of MISO downlink beamforming," IEEE Transactions on Communications, vol. 68, no. 3, pp. 1866-1880, 2019.

[76] A. Zappone, M. D. Renzo, and M. Debbah, "Wireless networks design in the era of deep learning: Model-based, AI-based, or both?” IEEE Transactions on Communications, vol. 67, no. 10, pp. 7331-7376, 2019.

[77] A. Balatsoukas-Stimming and C. Studer, "Deep unfolding for communications systems: A survey and some new directions," in IEEE International Workshop on Signal Processing Systems (SiPS). IEEE, 2019, pp. 266-271.

[78] T. V. Chien, T. N. Canh, E. Björnson, and E. G. Larsson, "Power control in cellular massive MIMO with varying user activity: A deep learning solution," IEEE Transactions on Wireless Communications, vol. 19, no. 9, pp. 5732-5748, 2020 .

[79] L. Sanguinetti, A. Zappone, and M. Debbah, "Deep learning power allocation in massive MIMO," in Proceedings of 52nd Asilomar Conference on Signals, Systems, and Computers. IEEE, 2018, pp. 1257-1261.

[80] M. Sadeghi and E. G. Larsson, "Adversarial attacks on deep-learning based radio signal classification," IEEE Wireless Communications Letters, vol. 8, no. 1, pp. 213-216, 2018. 
[81] T. Wang, C.-K. Wen, H. Wang, F. Gao, T. Jiang, and S. Jin, "Deep learning for wireless physical layer: Opportunities and challenges," China Communications, vol. 14, no. 11, pp. 92-111, 2017.

[82] Ö. T. Demir and E. Björnson, "Channel estimation under hardware impairments: Bayesian methods versus deep learning," in 16th International Symposium on Wireless Communication Systems (ISWCS). IEEE, 2019, pp. 193-197.

[83] E. Björnson and P. Giselsson, "Two applications of deep learning in the physical layer of communication systems [lecture notes]," IEEE Signal Processing Magazine, vol. 37, no. 5, pp. 134-140, 2020.

[84] G. Cybenko, "Approximation by superpositions of a sigmoidal function," Mathematics of control, signals and systems, vol. 2, no. 4, pp. 303-314, 1989. 



\section{Included Papers}




\section{Papers}

The papers associated with this thesis have been removed for copyright reasons. For more details about these see:

http://urn.kb.se/resolve?urn=urn:nbn:se:liu:diva-175777 




\section{Other Recently Published Theses From \\ The Division of Communication Systems \\ Department of Electrical Engineering (ISY) \\ Linköping University, Sweden}

Ziya Gülgün, Physical Layer Security Issues in Massive MIMO and GNSS, Linköping Studies in Science and Technology. Licentiate Thesis, No. 1899, 2021.

Giovanni Interdonato, Cell-Free Massive MIMO: Scalability, Signal Processing and Power Control, Linköping Studies in Science and Technology. Dissertations, No. 2090, 2020.

Özgecan Özdogan, Analysis of Cellular and Cell-Free Massive MIMO with Rician Fading, Linköping Studies in Science and Technology. Licentiate Thesis, No. 1870, 2020.

Ema Becirovic, On Massive MIMO for Massive Machine-Type Communications, Linköping Studies in Science and Technology. Licentiate Thesis, No. 1868, 2020.

Daniel Verenzuela, Exploring Alternative Massive MIMO Designs: Superimposed Pilots and Mixed-ADCs, Linköping Studies in Science and Technology. Dissertations, No. 2041, 2020.

Trinh Van Chien, Spatial Resource Allocation in Massive MIMO Communication: From Cellular to Cell-Free, Linköping Studies in Science and Technology. Dissertations, No. 2036, 2020 .

Marcus Karlsson, Blind Massive MIMO Base Stations: Downlink Transmission and Jamming, Linköping Studies in Science and Technology. Dissertations, No. 1950, 2018.

Victor Hei Cheng, Optimizing Massive MIMO: Precoder Design and Power Allocation, Linköping Studies in Science and Technology. Dissertations, No. 1929, 2018.

Christopher Mollén, High-End Performance with Low-End Hardware: Analysis of Massive MIMO Base Station Transceivers, Linköping Studies in Science and Technology. Dissertations, No. 1896, 2017.

Antonios Pitarokoilis, Phase Noise and Wideband Transmission in Massive MIMO, Linköping Studies in Science and Technology. Dissertations, No. 1756, 2016.

Anu Kalidas M. Pillai, Signal Reconstruction Algorithms for Time-Interleaved ADCs, Linköping Studies in Science and Technology. Dissertations, No. 1672, 2015.

Ngo Quoc Hien, Massive MIMO: Fundamentals and System Designs, Linköping Studies in Science and Technology. Dissertations, No. 1642, 2015. 
Mirsad Čirkić, Efficient MIMO Detection Methods, Linköping Studies in Science and Technology. Dissertations, No. 1570, 2014.

Reza Moosavi, Improving the Efficiency of Control Signaling in Wireless Multiple Access Systems, Linköping Studies in Science and Technology. Dissertations, No. 1556, 2014.

Tumula V. K. Chaitanya, HARQ Systems: Resource Allocation, Feedback Error Protection, and Bits-to-Symbol Mappings, Linköping Studies in Science and Technology. Dissertations, No. 1555, 2013. 


\title{
FACULTY OF SCIENCE AND ENGINEERING
}

\author{
Linköping Studies in Science and Technology, \\ Dissertation No. 2142, 2021 \\ Division of Communication Systems \\ of Electrical Engineering \\ Linköping University \\ SE-581 83 Linköping, Sweden \\ www.liu.se
}
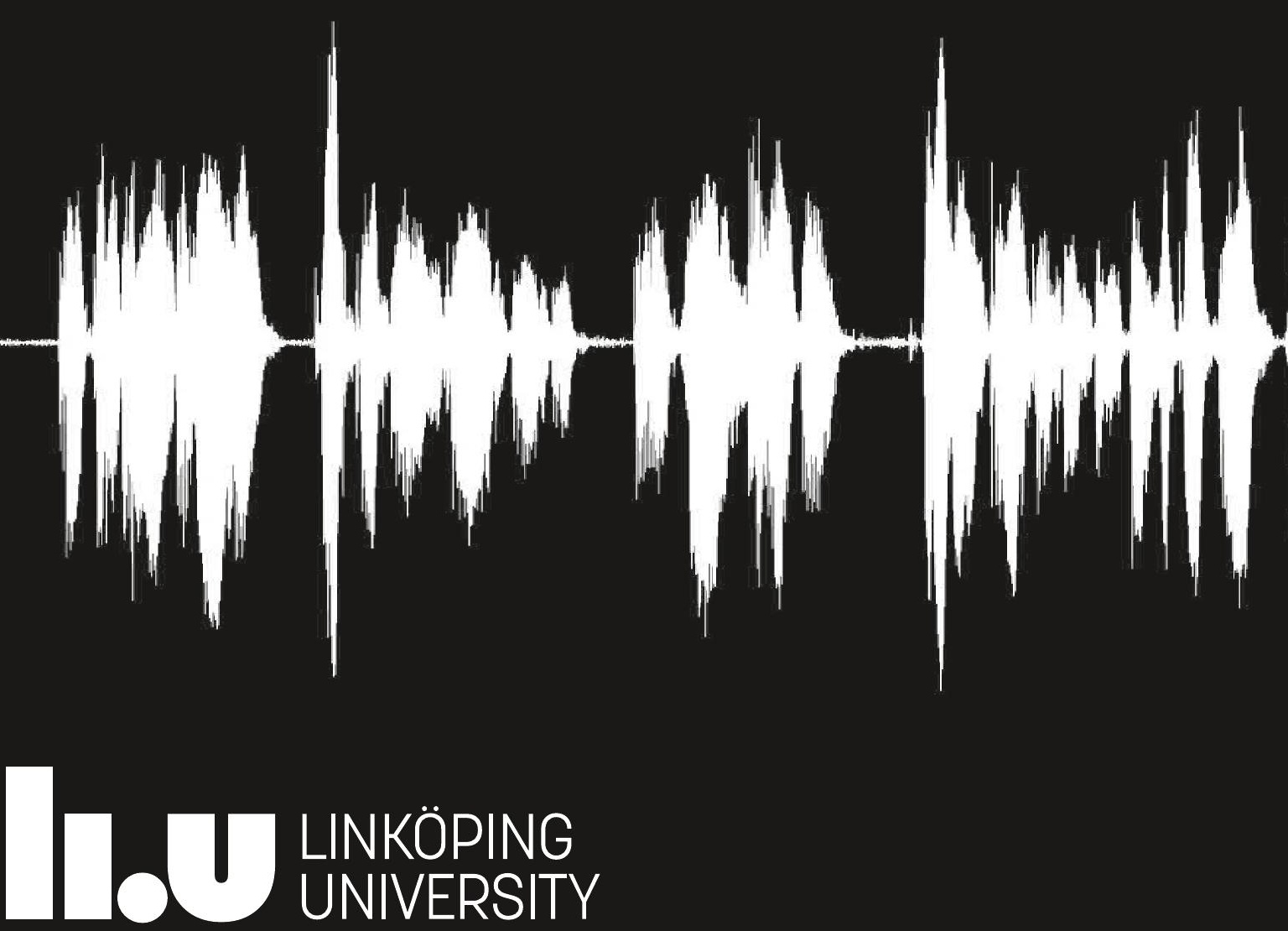